University of Nebraska - Lincoln

DigitalCommons@University of Nebraska - Lincoln

1991

Dynamical Approach Study of Spurious SteadyState Numerical Solutions of Nonlinear Differential Equations. I. The Dynamics of Time Discretization and Its Implications for Algorithm Development in Computational Fluid Dynamics

Helen C. Yee

NASA Ames Research Center, yee@nas.nasa.gov

P. K. Sweby

University of Reading

D. F. Griffiths

University of Dundee

Follow this and additional works at: http://digitalcommons.unl.edu/nasapub

Yee, Helen C.; Sweby, P. K.; and Griffiths, D. F., "Dynamical Approach Study of Spurious Steady-State Numerical Solutions of Nonlinear Differential Equations. I. The Dynamics of Time Discretization and Its Implications for Algorithm Development in Computational Fluid Dynamics" (1991). NASA Publications. 280.

http:// digitalcommons.unl.edu/nasapub/280

This Article is brought to you for free and open access by the National Aeronautics and Space Administration at DigitalCommons@University of Nebraska - Lincoln. It has been accepted for inclusion in NASA Publications by an authorized administrator of DigitalCommons@University of Nebraska - Lincoln. 


\title{
Dynamical Approach Study of Spurious Steady-State Numerical Solutions of Nonlinear Differential Equations. I. The Dynamics of Time Discretization and Its Implications for Algorithm Development in Computational Fluid Dynamics*
}

\author{
H. C. YeE
}

Fluid Dynamics Division, NASA Ames Research Center, Moffett Field, California 94035

P. K. SWEBY

Department of Mathematics, University of Reading, Whiteknights, Reading RG6 2AX, England

AND

D. F. GRIFFITHS

Department of Mathematical Sciences, University of Dundee, Dundee, DDI 4HN, Scotland

Received April 23, 1990; revised March 28, 1991

The goal of this paper is to utilize the theory of nonlinear dynamics approach to investigate the possible sources of errors and slow convergence and nonconvergence of steady-state numerical solutions when using the time-dependent approach for nonlinear hyperbolic and parabolic partial differential equations terms. This interdisciplinary research belongs to a subset of a new field of study in numerical analysis sometimes referred to as "the dynamics of numerics and the numerics of dynamics." At the present time, this new interdisciplinary topic is still the property of an isolated discipline with all too little effort spent in pointing out an underlying generality that could make it adaptable to diverse fields of applications. This is the first of a series of research papers under the same topic. Our hope is to reach researchers in the fields of computational fluid dynamics (CFD) and, in particular, hypersonic and combustion related CFD. By simple examples (in which the exact solutions of the governing equations are known), the application of the apparently straightforward numerical technique to genuinely nonlinear problems can be shown to lead to incorrect or misleading results. One striking phenomenon is that with the same initial data, the continuum and its discretized counterpart can asymptotically approach different stable solutions. This behavior is especially important for employing a time-dependent approach to the steady state since the initial data are usually not known and a freestream condition or an intelligent guess for the initial conditions is often used. With the unique property of the different dependence of the solution on initial data for the partial differential equation and the discretized counterpart, it is not easy to delineate the

* An abbreviated version appeared in the "Proceedings of the 12th International Conference on Numerical Methods in Fluid Dynamics, Oxford, England, July 9-13, 1990." The full text was published as an internal report-NASA Technical Memorandum 102820, April 1990. 
true physics from numerical artifacts when numerical methods are the sole source of solution procedure for the continuum. Part I concentrates on the dynamical behavior of time discretization for scalar nonlinear ordinary differential equations in order to motivate this new yet unconventional approach to algorithm development in CFD and to serve as an introduction for parts II and III of the same series of research papers. (C) 1991 Academic Press, Inc.

\section{INTRODUCTION}

During the late eighties, computer power and numerical algorithm development in computational fluid dynamics (CFD) advanced to a stage that allowed applied computational fluid dynamicists to channel their energy toward the modelling of more realistic three-dimensional (3D) complex aerodynamic configurations [1]. With the increased emphasis in recent years in high speed transports and advanced spacecraft design, algorithm developers in CFD have been faced with a new challenge. This stems from the fact that the majority of widely used numerical algorithms in CFD were originally designed to solve fluid flow problems that do not contain stiff nonlinear source terms, e.g., for perfect gas and equilibrium real gas flows. New algorithm and/or modification and improvement to existing numerical methods to accomodate the stiff nonlinear source term of nonhomogeneous hyperbolic and parabolic partial differential equations (PDEs) are urgently needed since nonequilibrium, combustion related, and certain turbulent models in Navier-Stokes gas dynamics problems are usually governed by PDEs of this type. Although much attention has been directed to improve the efficiency of existing numerical techniques and to extend existing Riemann solvers for nonequilibrium flows (cf. [2]), this comprises only a fraction of the required effort. The main difficulty lies in the basic understanding of genuinely nonlinear behavior of nonhomogeneous hyperbolic and parabolic PDEs and their discretized counterparts. The intent of this work is to gain some insight into the dynamics of numerics (the dynamical behavior of numerical schemes) for commonly used finite difference approximations in CFD. Here, to study the dynamical behavior of a numerical scheme means to study the local and global asymptotic behavior of the nonlinear difference equations resulting from finite discretizations of differential equations (DEs) subject to the variation of parameters such as the time step, grid spacing and numerical dissipation coefficient, etc. See Refs. [3-12] for an introduction to the theory of nonlinear dynamics and related subjects. Before presenting a brief background of the subject of nonlinear dynamics, a summary of our major findings including the results from our related papers in preparation [13-18] will be given.

\section{Summary of Results}

Utilizing the mathematical tools of nonlinear dynamics to analyze model nonlinear problems and problems containing nonlinear source terms, the following phenomena have been observed: (a) Stable and unstable spurious steady-state numerical solutions (numerically irrelevant solutions) can be independently 
introduced by commonly used spatial and temporal discretizations satisfying the same boundary condition and initial data (see Section IV and [16-18]). (b) Stable and unstable spurious steady-state numerical solutions can occur below as well as above the linearized stability limit of the schemes (see Section III and [13, 19, 20]). In other words, the result of operating with a time step below the linearized stability limit of the scheme does not necessarily result in a true approximation to the exact solution, and the result of operating with a time step above the linearized stability limit is not always a divergent solution. (c) There is a strong dependence of the numerical solution on initial data, analytical and numerical boundary conditions, and system parameters as well as the time step and grid spacing of the finite difference methods. It can be shown that with the same initial data, the continuum (PDEs or governing equations) and its discretized counterpart can asymptotically approach different stable solutions (see Section III). The unique property of the separate dependence of solutions on initial data for the individual continuum and its discretized counterparts is important for employing a timedependent approach to the steady state in fluid dynamics problems containing nonlinear source terms. In many CFD computations, the steady-state equations are PDEs of the mixed type and a time-dependent approach can avoid the complication of dealing with elliptic-parabolic or elliptic-hyperbolic types of PDEs. However, new uncertainty on the accuracy of the numerical solution arises. This uncertainty is due to the fact that the initial data are not known and a freestream condition or an intelligent guess for the initial conditions is often used. (d) The knowledge gained from the finite-difference method analysis for problems without nonlinear source terms does not carry over to the problems containing nonlinear source terms (see $[17,18,21$ ). (e) Spurious limit cycles can be generated by finite discretizations of nonlinear PDEs without source terms (see Section IV and [14]). (f) The existence of stable spurious limit cycles might be one of the contributing factors in nonconvergence of the time-dependent approach to the steady state (see Section III and [14]). We can also explain through a simple model reaction-convection boundary-value problem (BVP) the following phenomena. (g) The various ways of discretizing the reaction term can drastically affect the stability of the spurious as well as the exact steady-state solutions (see Section IV and [17, 18]). (h) The time discretization can destablize the stable spurious steady-state numerical solutions that are introduced by the spatial discretizations or vice versa (see Section IV and [16-18]). (i) The numerical phenomenon of incorrect propagation speeds of discontinuities [21] may be linked to the existence of some stable spurious steady-state numerical solutions (see Section IV and [17, 18]).

Although more theoretical development and existensive numerical experimentation are needed, we believe that these findings could have important implications in the interpretation of numerical results from existing computer codes and widely used CFD algorithms in combustion, reacting flows, and certain turbulence models in compressible Navier-Stokes computations. See Sections 2.3, 3.3, 3.4, and V for details.

At first glance, a few of these results might appear to have been discussed in some 
of the earlier numerical analysis and applied mathematics literature $[19,20$, 22-30]. However, our in-depth global and topographical approach to the problem and the discussion of the underlying implication and impact to the field of algorithm development in hypersonic CFD and combustion related computations are new. Although inspired in part by [27], the work of this series and our NASA internal reports $[31,13]$ was carried out independently from that of $[29,30]$.

\section{Nonlinear Dynamics and Chaotic Dynamics}

Before the birth of chaotic dynamical theory, traditional study of nonlinear dynamics belonged to the applied mechanics disciplines of mechanical engineering. Modern nonlinear dynamics (since the late seventies) includes chaotic dynamics. Strictly speaking, chaotic dynamics is a branch of nonlinear dynamics. But, for the purpose of the present discussion, unless otherwise stated, the term nonlinear dynamics and chaotic dynamics are used interchangeably. That is, nonlinear dynamics includes chaotic dynamics and vice versa.

Loosely speaking, the study of asymptotic behavior (and steady-state solutions) of nonlinear DEs and nonlinear discrete maps (difference equations) and how the asymptotes change as parameters of the system are varied is most often referred to as nonlinear dynamic analysis and chaotic dynamic theory. Topics in this area include bifurcation theory, period doubling cascades resulting in chaos, etc. Stable chaotic solutions (chaotic attractors) may be defined loosely and simply as stable asymptotes that have infinite period and yet are still bounded and are sensitive to initial data [3-11]. It is emphasized here that unless otherwise stated, all DEs and discrete maps are nonlinear and consist of system parameters, and the terms discrete maps and difference equations are used interchangeably.

\section{Types of Dynamical Systems}

Consider an ordinary differential equation (ODE) of the form

$$
\frac{d u}{d t}=\alpha S(u)
$$

where $\alpha$ is a parameter and $S$ is a nonlinear function of $u$ which is independent of $\alpha$. An ODE of this form in which $t$ does not appear explicitly in $S$ is called an autonomous dynamical system. One can also consider a function $S$ which is nonlinear in $u$ and depends explicitly on $t$. ODEs of this type are called nonautonomous dynamical systems and they are more difficult to analyze; see Refs. $[6,9]$ for a discussion. The analysis would be more complicated if $S=S(u, \alpha)$ is nonlinear in both $u$ and $\alpha$. In this case, the DE is not only nonlinear in the dependent variable $u$ (and independent variable $t$ ), but it is also nonlinear in the parameter space $\alpha$. One can also consider systems that depend on more than one parameter and/or systems of equations of the above type.

A PDE counterpart of (1.1) might be

$$
\frac{\partial u}{\partial t}+\frac{\partial f(u)}{\partial x}=\varepsilon \frac{\partial^{2} u}{\partial x^{2}}+\alpha S(u)
$$


where $\varepsilon$ is a parameter and the function $f(u)$ can be linear or nonlinear in $u$. The source term $S$ in (1.2) can be a nonlinear function similar to that of the ODE (1.1), except that $S$ can depend explicitly on $x$ as well as $t$ and $\alpha$.

Next consider nonlinear discrete maps (nonlinear difference equations) of the forms

$$
u^{n+1}=u^{n}+D\left(u^{n}, u^{n-1}, r\right)
$$

and

$$
u_{j}^{n+1}=u_{j}^{n}+G\left(u_{j}^{n}, u_{j \pm 1}^{n}, r\right) .
$$

Here $r$ is a parameter, and $D$ is nonlinear in $u^{n}$ and $u^{n-1}$ and linear or nonlinear in the parameter space $r$. The situation is similar for the function $G$. One can also consider discrete systems that depend on more than one parameter. A typical example is a discrete map arising from a finite-difference approximation of DEs such as (1.1) or (1.2). For the ODE, the resulting discrete maps might be nonlinear in $\alpha$ (even though the ODE is linear in $\alpha$ ) as well as the time step $\Delta t$, depending on the ODE solvers. For the PDE, again depending on the differencing scheme, the resulting discretized counterparts can be nonlinear in $\alpha, \Delta t$, the grid spacing $\Delta x$, and the numerical dissipation parameters even though the DEs consist of only one parameter or none. It is the introduction of new parameters due to the finite discretization that add a new dimension on the implication and interpretation of accuracy, stability, and convergence rate on asymptotic numerical solutions of DEs containing nonlinear source terms.

One can also consider discrete maps (scalar or system) of the forms

$$
u^{n+1}=u^{n}+D\left(u^{n+k}, \ldots, u^{n}, \ldots, u^{n-l}, r_{1}, r_{2}, \ldots, r_{m}\right)
$$

where $k, l, m$ are positive integers and $r_{1}, r_{2}, \ldots, r_{m}$ are parameters, and

$$
u_{j}^{n+1}=u_{j}^{n}+G\left(u_{j \pm 1}^{n+k}, \ldots, u_{j \pm 1}^{n}, \ldots, u_{j \pm 1}^{n-l}, u_{j}^{n+k}, \ldots, u_{j}^{n}, \ldots, u_{j}^{n-l}, r_{1}, r_{2}, \ldots, r_{m}\right) .
$$

Again, (1.6) can depend on more than the three indices $j, j \pm 1$. Systems (1.4) and (1.6) are sometimes referred to as partial-difference equations. Analysis of the dynamical behavior of (1.4) and (1.6) can be many orders of magnitude more difficult than that of (1.3) and (1.5). Any of the systems (1.1)-(1.6) are examples of dynamical systems. The discrete dynamical systems (discrete maps) (1.3)-(1.6) represent simple versions of what the CFD researchers are dealing with on a daily basis.

\section{Important Consideration}

It is emphasized here that discrete maps, regardless of their origin, are dynamical systems in their own right. It is also important to distinguish the following five types of discrete maps: 
1. Discrete maps arise naturally in physical sciences. They commonly arise through the inability to measure populations at all points in space and time $[6,11,32]$ in population dynamics. They can also arise through the study of periodic excitation of dynamical systems $[33,34]$ in applied mechanics.

2. Discrete maps arise from Poincare sections in ODEs [6, 9-11].

3. Discrete maps arise from discrete approximations of ODEs.

4. Discrete maps (partial-difference equations) arise from temporal and spatial finite difference approximations of PDEs.

5. Discrete models arise from the "inverse problems of nonlinear dynamics" in time series analysis of observable data or experiments [10].

Discrete maps of types 1 and 5 sometimes might not have any relationship with a specific DE. As a matter of fact, there might be no concrete associated governing equations (continuum or otherwise) to start with for type 5 except the surrogated discrete map arising from the time series analysis. Type 2 arises naturally from the study of dynamical behavior of nonlinear ODEs. However, types 3 and 4 have an intimate link (but with a different tie than type 2) between the original governing DEs and their discretized counterparts.

Note that for discrete maps of types 3 and 4, even though the DEs might be linear in the parameter space, depending on the numerical methods, the discretized counterparts might be linear or nonlinear in that parameter space. In addition, extra parameters which may appear linearly or nonlinear in the resulting discrete maps can also be introduced by the scheme as noted in the paragraph after Eq. (1.4). Furthermore, it is important to distinguish the complexity involved in the analysis of types 3 and 4 . Type 4 involves spatial as well as temporal dynamical behavior. That is, even though the PDE may not depend on any parameters at all, its discretized counterpart will depend (linearly or nonlinearly) on, e.g., the time step, grid spacing, and numerical dissipation coefficients in contrast to the ODE case, where fewer free parameters are involved. As can be seen in the subsequent sections, the nature of the dynamical behavior of these discrete maps is strongly influenced by properties of the numerical method and the types and forms of nonlinearity in the DEs. Moreover, when dealing with nonlinear conservation law PDEs, the dynamical behavior of the discretized counterparts is also strongly influenced by elements such as conservation and nonlinearity of the schemes and treatment of the source terms $[2,35-38,17.18]$. These issues are very crucial for the existence of spurious steady-state numerical solutions which will be explained in a later section. Here the term "nonlinear scheme" refers to a case where the resulting discretized counterparts are nonlinear when the scheme is applied to scalar constant coefficient linear PDEs [2].

\section{Objectives}

The study of the occurrence and the dynamical behavior of spurious steady-state numerical solutions for computational sciences applications is extremely difficult 
and complex to analyze and often unfamiliar to computational scientists as well as researchers working in nonlinear dynamics and nonlinear physics. A summary of the difficulty involved was discussed in Yee [2] and will be elaborated on in Sections IV and V. At present few general results are known. Our approach is first to gain an understanding of the dynamics of time discretization and then link this knowledge to the study of both the temporal and spatial nonlinear dynamical behavior of finite-difference methods for nonlinear PDEs of the nonhomogeneous hyperbolic and parabolic types.

This is the first of a series of research papers under the same topic. Yee and Sweby [14], the second of this series, is devoted to the study of the dynamics of numerics for $2 \times 2$ systems of ODEs. Lafon and Yee [17, 18], the third and fourth of this series, are devoted to the study of the possible errors, slow convergence, and nonconvergence of steady-state numerical solutions when using the time-dependent approach for a model nonlinear reaction-convection BVPs. In our companion papers $[13,16]$, we study the dynamical behavior of a class of explicit Runge-Kutta methods and related methods for PDEs. In another related paper [15], we study the dynamics of discrete traveling wave solutions of a nonlinear reaction-convection problem. The intent of this paper is not only to present a study of the state-of-the-art of nonlinear dynamical behavior of ODE solvers, but more importantly to serve as an introduction and to present new results to motivate this new yet unconventional concept to researchers in the field of CFD. Thus the mission of this paper is not to provide the answer or theory or to illustrate the connection of dynamical behavior of practical PDEs to their discretized counterparts, but rather to gain insight into the nonlinear features unconventional to this type of study and concentrate on the fundamentals. In order to bring out the new features, the illustrations concentrate on simple scalar DEs examples in which the exact solutions of the DEs are known.

\section{Outline}

The outline of the paper is as follows: First, a brief background, motivation, and basic ideas will be given. Then some typical characteristics of dynamical systems with genuinely nonlinear behavior will be discussed. Next, the dynamical behavior of discrete maps arising from time discretization of ODEs will be studied and the main results and their implications for computational sciences will be described. Studies on discrete maps arising from finite-difference approximations of PDEs will not be elaborated. Rather, the level of complexity involved and state-of-the-art study on this subject will be briefly described. Remarks will be given on the popular misconception of residual test for convergence in steady-state solution via the "time-dependent" approach and the popular misconception of the use of the "inverse problems of nonlinear dynamics" to analyze the dynamical behavior of time series data from a computer code in an attempt to learn about the true behavior of the solution of the governing PDEs. This application of time series analysis can be misleading, and a wrong conclusion can be reached if the 
practitioner does not know by other means the exact solution behavior of the PDEs other than that given by the numerical solutions. The paper will conclude with a few recommendations.

\section{Motivation AND Relevance}

As discussed in the introduction, dynamical systems occur in the form of DEs and discrete maps. In order to motivate why the study of numerical analysis will not be complete without the utilization of the nonlinear dynamics approach and to convey to practitioners in computational sciences the importance of distinguishing between weakly nonlinear problems and genuinely nonlinear problems, this section is devoted to a discussion of dynamical systems with genuinely nonlinear behavior and the basic difference in dynamical behavior between DEs and discrete maps. This discussion leads to the key elements of this paper, namely: (1) to establish the connection between the DEs and their discretized counterparts and (2) to convey to computational scientists how traditional ways of thinking and conventional practices must change when dealing with genuinely nonlinear problems.

\subsection{Typical Characteristics of Dynamical Systems with Genuinely Nonlinear Behavior}

The terms "nonlinear behavior" and "genuinely nonlinear behavior" are used quite often in the literature and there seems to be no unified exact definition or meaning [10]. Here these terms are used for nonlinear dynamical systems that exhibit mainly the following characteristics:

(1) The study of nonlinear dynamics most often emphasizes the importance of obtaining a global qualitative understanding of the character of the system's dynamics, since local analysis is not sufficient to give the global behavior of genuinely nonlinear dynamical systems. As a matter of fact, this is one of the major reasons why sometimes the study of genuinely nonlinear dynamical systems required orders of magnitude more work than solving their linear counterparts.

(2) Unlike the situation for linear or weakly nonlinear problems, the solutions of genuinely nonlinear DEs and discrete maps are strongly dependent on system parameters, initial data, and boundary conditions.

(3) Only genuinely nonlinear dynamical systems can have chaotic behavior and one of the striking characteristics of chaotic behavior is sensitivity of the solution to initial data. This characteristic is independent of whether the dynamical system is a continuum or a discrete map.

\subsection{Typical Difference in Dynamical Behavior of ODEs and Discrete Maps}

The study of discrete maps is the discrete analog to the study of ODEs, as the study of recursion formulas is a discrete analog to the study of series expansions of 
functions. Much of the theory of ODEs can carry over to discrete maps with some slight modifications. However, there are new phenomena occurring in discrete maps which are absent in differential systems [39, 40, 33, 34].

With respect to the topographical behavior, there are new kinds of behaviors of trajectories in the neighborhood of fixed points (to be defined in Section III) of discrete maps. The behavior of separatrices associated with a saddle type of fixed point for a nonlinear difference system is far more complicated than the behavior of separatrices for the corresponding differential system. See Yee, Hsu, and Hsu et al. $[33,34,41,42]$ for details and examples.

With respect to similar equation types, the minimum number of first-order nonlinear autonomous ODEs is three for the existence of chaotic phenomena. However, a simple scalar first-order difference equation like the logistic map [43-47]

$$
v^{n+1}=\mu v^{n}\left(1-\frac{v^{n}}{4}\right), \quad \mu \text { a parameter, }
$$

or its piecewise linear approximation [48],

$$
\begin{aligned}
v^{n+1} & =\mu v^{n}, & & v^{n} \leqslant 1 \\
& =\mu, & & 1 \leqslant v^{n} \leqslant 3 \\
& =\mu\left(4-v^{n}\right), & & 3 \leqslant v^{n},
\end{aligned}
$$

possesses very rich dynamical behavior such as period-doubling cascades resulting in chaos. Equation (2.2) has the same behavior as (2.1) except that simple closed form asymptotic solutions of all periods can be obtained. These characteristic trait differences between ODEs and discrete maps are very general. The discrete maps can arise from any of the five types as discussed in the introduction. It is in this spirit that we say that discrete maps can exhibit a much richer range of dynamical behavior than DEs. The next two sections focus on the typical difference and connection between the dynamical behavior of ODEs and their discretized counterparts.

\subsection{Background and Motivation}

Spurious asymptotic numerical solutions such as chaos were observed by Ushiki [49] and Brezzi et al. [50] when they used the leapfrog method of discretization for the logistic ODE

$$
\frac{d u}{d t}=\alpha u(1-u)
$$

In Ref. [22], Schreiber and Keller discussed the existence of spurious asymptotic numerical solutions for a driven cavity problem described by an elliptic PDE. Some 
related studies are reported in [23]. Newell [19] gave a detailed account on the local behavior of finite amplitude instabilities of partial difference equations.

Spurious solutions of Burgers' equation and channel flows have been studied and computed in [24-26]. Their main emphasis is on the dynamics of numerics for some steady-state PDEs. Many other investigators in the computational sciences (e.g., [51-55]) have observed some kind of strange or chaotic behavior introduced by the numerical methods, but were not able to explain precisely the overall connection and differences between the continuum and its discretized counterpart, or most of all the implication and impact in practical applications in computational sciences.

In the early and mid eighties, it had been realized by numerical analysts that discrete maps resulting from finite discretization of ODEs and PDEs can be considered as dynamical systems. Several papers (see, e.g., [56,57]) on numerical methods as dynamical systems have appeared in recent years. These investigators studied the dynamical behavior of the different ODE solvers per se without relating their close tie with the ODEs themselves. Although the study of chaotic dynamics for nonlinear differential equations and for discrete maps have independently flourished for the last decade, there are very few investigators addressing the issue of the connection between the nonlinear dynamical behavior of the continuous systems and the corresponding discrete map resulting from finite difference discretizations. This issue is especially vital for computational sciences since nonlinear differential equations in applied sciences can rarely be solved in closed form and it is often necessary to replace them by finite dimensional nonlinear discrete maps. Most often, typical applied scientists rely on numerical methods to give insight into the solution behavior of nonlinear DEs. It is not always clear how well a numerical solution can mimic the true physics of problems that possess genuinely nonlinear types of behavior.

Why is there such a need to study the connection between the dynamical behavior of the continuum and its discretized counterparts for CFD applications? As indicated at the beginning of Section I, the major reason is that existing widely used numerical algorithms in CFD were originally designed to solve fluid flow problems that do not contain stiff nonlinear source terms. A straightforward application of these numerical methods to nonequilibrium flow or combustion related model problems can lead to wrong result, slow convergence, or even nonconvergent solutions. See later sections and $[17,18]$ for details. Another necessity stems from the fact that current supercomputer power can perform numerical simulations on virtually any simple $3 \mathrm{D}$ aerodynamic configuration and, due to the limited available experimental data, some of the applied engineers are forced to rely on the numerical simulations to help design the next generation of aircraft and spacecraft. However, many of these applied scientists are still using linearized analysis as their guide to studying nonhomogeneous hyperbolic and parabolic PDEs with nonlinear source terms, and often they are not aware of the limitations and pitfalls of the numerical procedures. For example, it is a common practice in CFD that the exact linearized stability limit is usually not computed, but rather a 
frozen coefficient procedure at each time step with a fixed grid spacing is used to estimate the stability limit of the algorithm. As can be seen later, the use of this type of linearized analysis guideline for genuinely nonlinear problems such as nonequilibrium flows can lead to misleading results.

\subsection{Connection between the Dynamical Behavior of the Continuum and Its Discretized Counterpart}

Aside from truncation error and machine round-off error, a more fundamental distinction between the continuum and its discretized counterparts is new behavior in the form of stable and unstable spurious asymptotes created by the numerical methods. This is due to the fact that nonlinear discrete maps can exhibit a much richer range of dynamical behavior than their continuum counterparts as discussed in Section 2.2. Some instructive examples will be given in Section III. These new phenomena were partially explored by the University of Dundee group [58-66], Sanz Serna and Vadillo [67], Iserles [27-29] and Stuart [20, 68-71]. Their main emphasis was on phenomena beyond the linearized stability limit. Study of the local existence of unstable spurious steady-state numerical solutions and stable spurious asymptotes of higher than period 1 below the linearized stability limit was observed by Newell [19] and Stuart [20]. Adams et al. [72] discussed spurious chaotic phenomena in astrophysics and celestial mechanics. Adams [73, 74] also discussed the use of interval arithmatics (interval mathematics or enclosure methods) to approach the dynamics of numerics. Moore et al. [75] discussed the reliability of numerical experiments in thermosolutal convection. Keener [76] discussed the uses and abuses of numerical methods in cardiology. The main contributions of our current study (including our related papers [13-18]) are (1) the detailed global and topographical approach to the occurrence of stable and unstable spurious steadystate numerical solutions below as well as above the linearized stability limit of the scheme for genuinely nonlinear problems, (2) the link of the strong dependence of numerical solutions on the time step, grid spacing, initial data, analytical and numerical boundary conditions, numerical dissipation terms, and basins of attraction to the time dependent approach to steady-state numerical solutions, and (3) the implications for practical computations in combustion and hypersonic CFD.

Before the numerical examples are discussed, an overall summary of our curent findings (integrated with other relevant recent results) will be given in the next two subsections. The discussion is devoted first to steady-state solutions and asymptotes of any period, and second to transient solutions.

\subsubsection{Steady-State Solutions and Asymptotes of Period Higher than One}

Table I shows a comparison of the possible stable asymptotic solution behavior between DEs (ODEs or PDEs) and their discretized counterparts. Some of the phenomena will be supported by simple examples in Section III and our companion papers [13-18]. The main connection between the DEs and their discretized counterparts is that steady-state solutions of the continuum are usually solutions of the 
TABLE I

Possible Stable Asymptotic Solution Behavior for DEs and Their Discretized Counterparts

\begin{tabular}{lcc}
\hline $\begin{array}{c}\text { Solution } \\
\text { type }\end{array}$ & ODEs or PDEs & $\begin{array}{c}\text { Discretized } \\
\text { Counterparts }\end{array}$ \\
\hline Steady-state solutions & Single & Single \\
& Single & Multiple \\
& Multiple & Same no. of multiple \\
Multiple & Additional no. of multiple \\
Periodic solutions & No & Yes \\
& Yes & Yes (+ extra) \\
Chaos & No & Yes \\
& Yes & Yes (+extra) \\
\hline
\end{tabular}

discretized counterparts (assume the schemes are consistent) but not the reverse. Their main difference is that new phenomena can be introduced by the numerical methods in the form of stable and unstable spurious asymptotic solutions of any period. This stems from the fact that even though, e.g., we start with a scalar firstorder nonlinear autonomous ODE (and thus the dynamics of a 1D autonomous ODE behavior), its discretized counterpart can have the dynamics of up to an infinite dimensional dynamical system behavior (due to the discrete recursive relationship of the nonlinear map). The situation is more complicated for multi-stage methods such as the Runge-Kutta method of order higher than one. As oppose to linear multistep methods (LMMs), the Runge-Kutta type of methods can introduce nonlinearity (in the parameter space $\Delta t$ ) to the resulting discretized equation (for DEs containing nonlinear source terms).

In the past, phenomena of spurious asymptotes were observed largely beyond the linearized stability limit of the scheme. Some numerical analysts and applied computational scientists rather than being alarmed were skeptical about these phenomena since, theoretically, one is always guided by the linearized stability limit of the scheme. However, this reasoning is only valid if one is solving a scalar nonlinear ODE using a variable step size control and known initial data. Another important concept is that the result of operating with time steps beyond the linearized stability limit is not always a divergent solution; spurious steady-state solutions and spurious asymptotes of higher period can occur.

Additionally, our current study indicated that, depending on the form of the nonlinear DEs, all ODE solvers can introduce stable and unstable spurious asymptotic solutions of some period or all periods. But, the most striking result is that for certain schemes, and depending on the form of the nonlinear DEs, stable spurious steady states can occur below the linearized stability limit. See Section III and our companion paper [13] for more details.

Another important concept is the interplay between initial data, spurious asymptotes, basins of attraction, and the time dependent approach to the steady- 


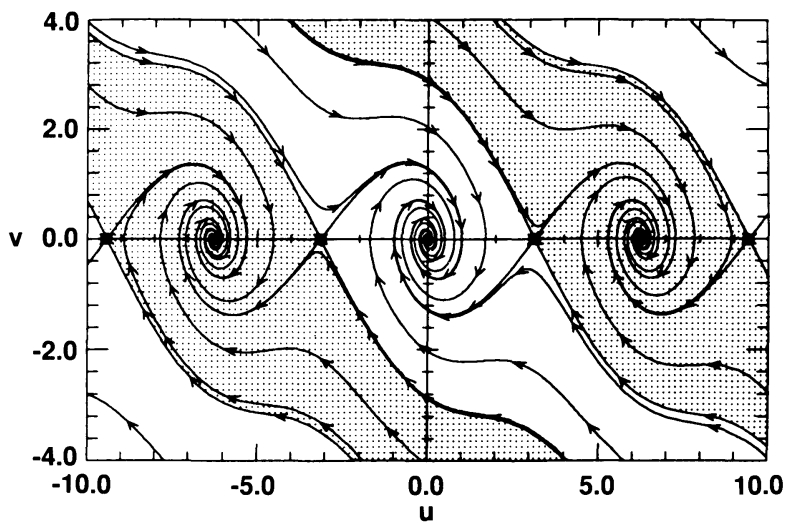

Fig. 2.1. Phase portrait and basins of attraction of the damped pendulum equation (this figure is taken from Ref. [9]).

state numerical solutions. Here the basin of attraction of a dynamical system is the domain of the set of initial conditions whose solution curves (trajectories) all approach the same asymptotic state. Figures 2.1 and 2.2 show the basins of attraction of two popular ODE dynamical systems. Figure 2.1 shows the multiple stable steady states and their basins of attraction for the damped pendulum equation

$$
\begin{aligned}
& \frac{d u}{d t}=v, \\
& \frac{d v}{d t}=-\varepsilon v-\sin (u)
\end{aligned}
$$

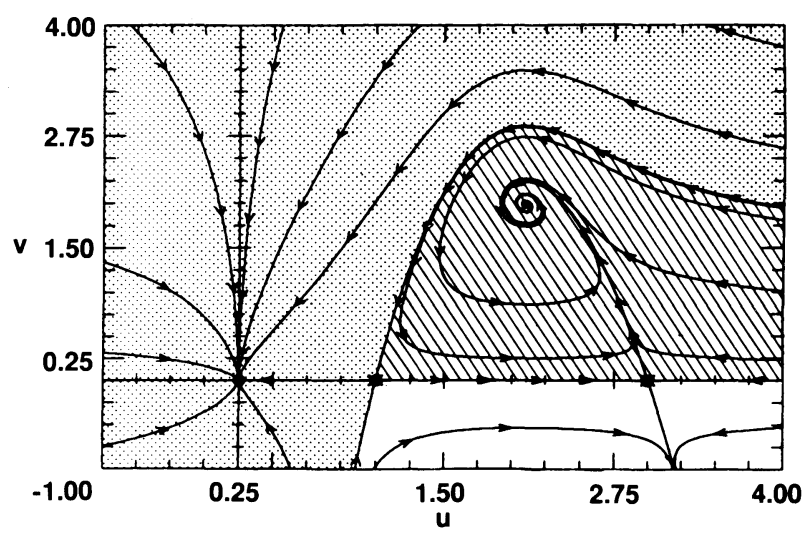

FIG. 2.2. Phase portrait and basins of attraction of the predator-prey equation (this figure is taken from Ref. [9]). 
for $\varepsilon=0.4$. Figure 2.2 shows the multiple steady states and their basins of attraction for the simple predator-prey equation

$$
\begin{aligned}
& \frac{d u}{d t}=-3 u+4 u^{2}-u v / 2-u^{3}, \\
& \frac{d v}{d t}=-2.1 v+u v,
\end{aligned}
$$

where $u$ is the population of the prey and $v$ is the population of the predator. These figures are taken from Parker and Chua [9] and were generated by the use of a variable time step Runge-Kutta-Fehlberg method with a built-in accuracy check (roughly speaking, a check whether the numerical asymptote satisfies the righthand side of (2.4) or (2.5)). See Ref. [9] for details. Although generated numerically, nevertheless the basins of attraction and fixed points of these figures coincide with those of exact solutions of the ODEs, owing to the built-in accuracy check. The stable fixed points of the damped pendulum equation are $2 n \pi, n=0,1, \ldots$. The unstable fixed points (saddles) are $(2 n+1) \pi$. The separatrices of the saddle points divide the phase plane into the different basins of attraction for the corresponding stable fixed points. The fixed points of the predator and prey equation are slightly less regular than those for the damped pendulum equation. Figure 2.2 shows two saddle points at $u=1, v=0$ and $u=3, v=0$, one stable focal point at $u=2.1$, $v=1.98$, and one stable nodal point at $u=0, v=0$. Again the separatrices of the saddle points divide the phase plane into the basins of attraction for the corresponding stable fixed points.

Contrary to the Runge-Kutta-Fehlberg method with a built-in accuracy check, some explicit Runge-Kutta methods with fixed time steps can produce spurious limit cycles (isolated periodic orbits [6]). See our companion paper [14] for a discussion. One can visualize the difference in the basins of attraction between Figs. 2.1 and 2.2 and the corresponding ones that are generated by some of the explicit Runge-Kutta methods. Intuitively, in the presence of spurious asymptotes (introduced by the numerical methods), the basin of the true stable steady states (steady states of the DEs) can be separated by the basins of attraction of the stable spurious asymptotes and interwoven by unstable asymptotes, whether due to the physics (i.e., present in both the DEs and the discretized counterparts) or spurious in nature (i.e., introduced by the numerical methods). That is, associated with the same (common) steady-state solution, the basin of attraction (domain of attraction) of the continuum might be very different from the discretized counterparts. This is due entirely to the different dependence on and sensitivity to initial and boundary conditions for the individual system. The situation is compounded by the existence of spurious steady states and asymptotes of period higher than one, and possibly chaotic attractors.

This unique dynamical property of the different dependence of solutions on initial data for the individual nonlinear DE and its discretized counterpart is especially 
important for employing a "time-dependent" approach to the steady state with given initial data in hypersonic CFD. In many CFD computations, the steady-state equations are PDEs of the mixed type and a time-dependent approach to the steady state can avoid the complication of dealing with elliptic-parabolic or elliptic-hyperbolic types of PDEs. However, this time-dependent approach has created a new dimension of uncertainty. This uncertainty stems from the fact that in practical computations, the initial data are not known and a freestream condition or an intelligent guess for the initial conditions is used. In particular, the controversy of the "existence of multiple steady-state solutions" through numerical experiments [77] will not be resolved until there is a better understanding of the different dependence on initial data for both the PDEs and the discretized equations.

For PDEs, there is an additional difficulty in that even with the same time discretization but different spatial discretizations or vice versa, the basins of attraction can also be extremely different. However, mapping out the basins of attraction for any nonlinear continuum dynamical system other than the very simple scalar equations relies on numerical methods. The type of nonlinear behavior and the dependence and sensitivity to initial conditions for both the PDEs and their discretized counterparts make the understanding of the true physics extremely difficult when numerical methods are the sole source. Under this situation, how can one delineate the numerical solutions that approximate the true physics from the numerical solutions that are spurious in nature? Hopefully, with our simple illustrations in Section III, we can demonstrate the importance of the subject and, most of all, the importance of knowing the general dynamical behavior of asymptotes of the schemes for genuinely nonlinear scalar DEs before applying these schemes in practical computations.

Due to the popularity of searching for chaotic phenomena, it is very trendy to relate inaccuracy in numerical methods with the onset of "numerical chaos." It is emphasized here that unless one is searching for chaotic phenomena, inaccuracy in long time integration of discrete maps resulting from finite discretization of nonlinear DEs comes in other forms prior to the onset of numerical chaos. Stable and unstable spurious steady states and spurious periodic numerical solutions set in before numerical chaos occurs. These spurious asymptotes of finite period are just as inaccurate as numerical chaos as far as numerical integration is concerned. In other words, the prelude to numerical chaos is the key element that we want to stress (i.e., before the the onset of chaos or a divergent solution). As can be seen in a later section, this behavior is more difficult to detect than numerical chaos in practical computations.

\subsubsection{Transient or Time-Accurate Solutions}

It is a common misconception that inaccuracy in long-time behavior poses no consequences on transient or time-accurate solutions. This is not the case when one is dealing with genuinely nonlinear DEs. For genuinely nonlinear problems, due to the possible existence of spurious solutions, larger numerical errors can be introduced by the numerical methods than one can expect from local linearized analysis 
or weakly nonlinear behavior. The situation will get more intensified if the initial data of the DE is in the basin of attraction of a chaotic transient [78-80] of the discretized counterpart. This is due to the fact that existence of spurious asymptotes and/or chaotic transient transact the wrong behavior in finite time. In fact, it is possible the whole solution trajectory is erroneous.

\section{The Numerical ODE CONNECTION}

In this section, we review some of the fundamentals and available theory and discuss our major results. The discussion will have some overlap with our companion paper [13].

\subsection{Preliminaries}

Consider an autonomous nonlinear ODE of the form

$$
\frac{d u}{d t}=\alpha S(u)
$$

where $\alpha$ is a parameter and $S(u)$ is nonlinear in $u$. For simplicity of discussion, we consider only autonomous ODEs, where $\alpha$ is linear in (3.1); i.e., $\alpha$ does not appear explicitly in $S$.

A fixed point $u^{*}$ of an autonomous system (3.1) is a constant solution of (3.1); that is,

$$
S\left(u^{*}\right)=0 .
$$

Note that the terms "equilibrium points," "critical points," "singular points," "stationary points," "asymptotic solutions" (we are excluding periodic solutions for the current definition), "steady-state solutions," and "fixed points" are sometimes used with slightly different meanings in the literature, e.g., in bifurcation theory. For the current discussion and for the majority of the nonlinear dynamics literature, these terms are used interchangeably. Note that certain researchers reserve the term "fixed point" for discrete maps only.

Consider a nonlinear discrete map from finite discretization of (3.1),

$$
u^{n+1}=u^{n}+D\left(u^{n}, r\right),
$$

where $r=\alpha \Delta t$ and $D\left(u^{n}, r\right)$ is linear or nonlinear in $r$ depending on the ODE solver. Here the analysis is similar if $D$ is a nonlinear function of $u^{n+p}, p=0,1, \ldots, m$. Examples to illustrate the dependence on the numerical schemes for cases where $D$ is linear or nonlinear in the parameter space will be given in the subsequent section.

A fixed point $u^{*}$ of (3.3) (or fixed point of period 1) is defined by $u^{n+1}=u^{n}$, or

$$
u^{*}=u^{*}+D\left(u^{*}, r\right)
$$


or

$$
D\left(u^{*}, r\right)=0 .
$$

One can also define a fixed point of period $p$, where $p$ is a positive integer by requiring that $u^{n+p}=u^{n}$ and

$$
u^{*}=E^{p}\left(u^{*}, r\right) \quad \text { but } \quad u^{*} \neq E^{k}\left(u^{*}, r\right) \quad \text { for } 0<k<p .
$$

Here, $E^{p}\left(u^{*}, r\right)$ means that we apply the difference operator $E p$ times, where $E\left(u^{n}, r\right)=u^{n}+D\left(u^{n}, r\right)$. For example, a fixed point of period 2 means $u^{n+2}=u^{n}$ or

$$
u^{*}=E\left(E\left(u^{*}, r\right)\right)
$$

In the context of discrete systems, the term "fixed point" without indicating the period means "fixed point of period 1" or the steady-state solution of (3.3).

Note that Eq. (3.6) is equivalent to a $2 \times 2$ first-order nonlinear discrete map and Eq. (3.5) is equivalent to a $p \times p$ first-order nonlinear discrete map. Although the continuum has the behavior of a first-order ODE, due to the the nature of the recursive relationship and the type and order of the ODE solvers, the various asymptotes of the discretized counterpart have the dynamics of higher and even infinite dimensional dynamical system behavior. It is this crucial factor that induces richness into the dynamics of numerics and the numerics of dynamics.

In order to illustrate the basic idea, the simplest form of the Ricatti ODE, i.e., the logistic ODE (2.3) with

$$
S(u)=u(1-u)
$$

is considered. For this ODE, the exact solution is

$$
u(t)=\frac{u^{0}}{u^{0}+\left(1-u^{0}\right) e^{-x t}},
$$

where $u^{0}$ is the initial condition. The fixed points of the logistic equation are roots of $u^{*}\left(1-u^{*}\right)=0$; it has two fixed points $u^{*}=1$ and $u^{*}=0$.

To study the stability of these fixed points, we perturb the fixed point with a disturbance $\xi$ and obtain the perturbed equation

$$
\frac{d \xi}{d t}=\alpha S\left(u^{*}+\xi\right)
$$

Next, $S\left(u^{*}+\xi\right)$ can be expanded in a Taylor series around $u^{*}$, so that

$$
\frac{d \xi}{d t}=\alpha\left[S\left(u^{*}\right)+S_{u}\left(u^{*}\right) \xi+\frac{1}{2} S_{u u}\left(u^{*}\right) \xi^{2}+\cdots\right],
$$


where $S_{u}\left(u^{*}\right)=d S /\left.d u\right|_{u^{*}}$. Stability can be detected by examining a small neighborhood of the fixed point provided if, for a given $\alpha, u^{*}$ is not a hyperbolic point [3-6, 8-11] (i.e., if the real part of $\alpha S_{u}\left(u^{*}\right) \neq 0$ ). Under this condition $\xi$ can be assumed small, its successive powers $\xi^{2}, \xi^{3}, \ldots$ can normally be neglected, and the following linear perturbed equation is obtained:

$$
\frac{d \xi}{d t}=\alpha S_{u}\left(u^{*}\right) \xi
$$

The fixed point $u^{*}$ is asymptotically stable if $\alpha S_{u}\left(u^{*}\right)<0$, whereas $u^{*}$ is unstable if $\alpha S_{u}\left(u^{*}\right)>0$. If $\alpha S_{u}\left(u^{*}\right)=0$, a higher order perturbation is necessary.

If we perturb the logistic equation around the fixed point with $\alpha>0$, we find that $u^{*}=1$ is stable and $u^{*}=0$ is unstable. It is well known that the global asymptotic solution behavior of the logistic ODE is that for any $u^{0}>0$, the solution will eventually tend to $u^{*}=1$. Figure 3.1 shows the asymptotic solution behavior of the logistic ODE.

Now, let us look at three of the well-known ODE solvers. These are explicit Euler (Euler, forward Euler), leapfrog, and Adam-Bashforth. For the ODE (3.1) with $S(u)=u(1-u)$, the dynamical behavior of their corresponding discrete maps is well established. The explicit Euler method is given by

$$
u^{n+1}=u^{n}+r S\left(u^{n}\right)
$$

and after a linear transformation, it is the well known logistic map [43-47]. The leapfrog method can be written as

$$
u^{n+1}=u^{n-1}+2 r S\left(u^{n}\right),
$$

and it is a form of the Henon map [49]. The Adam-Bashforth method yields

$$
u^{n+1}=u^{n}+\frac{r}{2}\left[3 S\left(u^{n}\right)-S\left(u^{n-1}\right)\right]
$$

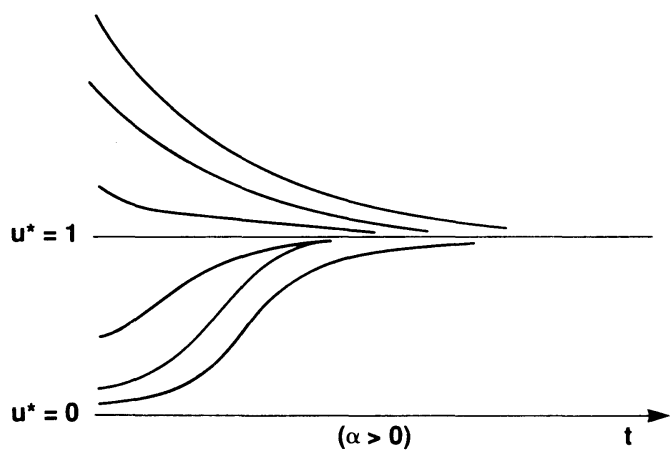

FIG. 3.1. Asymptotic solution behavior of the logistic ODE $d u / d t=\alpha u(1-u)$ for $\alpha>0$. 
which is again a variant of the Henon map that has been discussed by Pruffer [56] in detail.

We can determine fixed points of the discrete maps (3.12)-(3.14) and their stability properties in a manner similar to that for the ODE. It turns out that all three of the discrete maps have the same fixed points as the ODE (3.1) - a desired property which is important for obtaining asymptotes of nonlinear DEs numerically. Here we use the term asymptote to mean a fixed point of any period.

The corresponding linear perturbed equation for the discrete map (3.3), found by substituting $u^{n}=u^{*}+\xi^{n}$ in (3.3) and ignoring terms higher than $\xi^{n}$, is

$$
\xi^{n+1}=\xi^{n}\left[1+\Delta t D_{u}\left(u^{*}, \Delta t\right)\right] .
$$

Here the parameter $\alpha$ of the ODE has been absorbed in the parameter $\Delta t$ based on the assumption that $\alpha$ does not appear explicitly in $S(u)$. Depending on the ODE solvers, $D\left(u^{n}, \Delta t\right)$ might be nonlinear in $\Delta t$. The possibility of nonlinearity in the parameter space $\Delta t$ being introduced into the discretized counterpart makes the dynamics of numerics deviate from the dynamics of the continuum. See Section 3.4 for details. For stability we require

$$
\left|1+\Delta t D_{u}\left(u^{*}, \Delta t\right)\right|<1 .
$$

Again, for $\left|1+\Delta t D_{u}\left(u^{*}, \Delta t\right)\right|=1$, higher order perturbation is necessary. For a fixed point of period $p$ the corresponding linear perturbed equation and stability criterion $[3-6]$ are

$$
\xi^{n+p}=\xi^{n} E_{u}^{p}\left(u^{*}, \Delta t\right)
$$

and

$$
\left|E_{u}^{p}\left(u^{*}, \Delta t\right)\right|<1,
$$

with

$$
E_{u}^{p}\left(u^{n}, \Delta t\right)=\frac{d}{d u} E\left(u^{n+p-1}, \Delta t\right) \cdots \frac{d}{d u} E\left(u^{n}, \Delta t\right) .
$$

For $S(u)=u(1-u)$, the stability of the stable fixed points of periods 1 and 2 for discrete maps (3.12)-(3.14) with $r=\alpha \Delta t$ are

Explicit Euler.

$$
\begin{array}{ll}
u^{*}=1 & \text { stable if } 0<r<2 \\
\text { period } 2 & \text { stable if } 2<r<\sqrt{6} .
\end{array}
$$

Leapfrog.

$$
\begin{aligned}
& u^{*}=1 \quad \text { unstable for all } r \geqslant 0 \\
& \text { chaotic solutions exist for all } r \text { no matter how small. }
\end{aligned}
$$


Adam-Bashforth.

$$
\begin{array}{ll}
u^{*}=1 & \text { stable if } 0<r<1 . \\
\text { period } 2 & \text { stable if } 1<r<\sqrt{2} .
\end{array}
$$

Figure 3.2a shows the stable fixed point diagram of periods 1, 2, 4, 8 obtained for the explicit Euler scheme by solving numerically the roots of (3.12) (by setting $\left.u^{n+1}=u^{n}\right)$ for $S(u)=u(1-u)$. The $r$ axis is divided into 1000 equal intervals. The numeric labelling of the branches denotes their period. The subscript $E$ on the period 1 branch indicates the stable fixed point of the DE.

Two of these three examples serve to illustrate that the result of operating with a time step beyond the linearized stability limit of the stable fixed points of the nonlinear ODEs is not always a divergent solution; spurious asymptotes of higher period can occur. This is in contrast to the ODE solution, where only a single stable asymptotic value $u^{*}=1$ exists for any $\alpha>0$ and any initial data $u^{0}>0$. It is emphasized here that these spurious asymptotes, regardless of the period, stable or unstable, are solutions in their own right of the discrete maps resulting from a finite discretization of the ODE.

\subsection{Spurious Steady-State Numerical Solutions}

For the previous three ODE solvers, we purposely picked the type of schemes that do not exhibit spurious fixed points [27] but allow spurious fixed points of period higher than 1 . In this section, we discuss the existence of spurious steadystate numerical solutions. Again, it is emphasized here that these spurious steady states, stable or unstable, are solutions in their own right of the resulting discrete maps. Consider two second-order Runge-Kutta schemes, namely, the modified Euler (R-K 2) and the improved Euler (R-K 2), the fourth-order Runge-Kutta method (R-K 4), and the second and third-order predictor-corrector method [81-83] of the forms:

Modified Euler (R-K 2) method,

$$
u^{n+1}=u^{n}+r S\left(u^{n}+\frac{r}{2} S^{n}\right), \quad S^{n}=S\left(u^{n}\right) .
$$

Improved Euler (R-K 2) method,

$$
u^{n+1}=u^{n}+\frac{r}{2}\left[S^{n}+S\left(u^{n}+r S^{n}\right)\right] .
$$

FIG. 3.2. Stable fixed points of periods $1,2,4,8$ for the logistic ODE $d u / d t=\alpha u(1-u)$. 

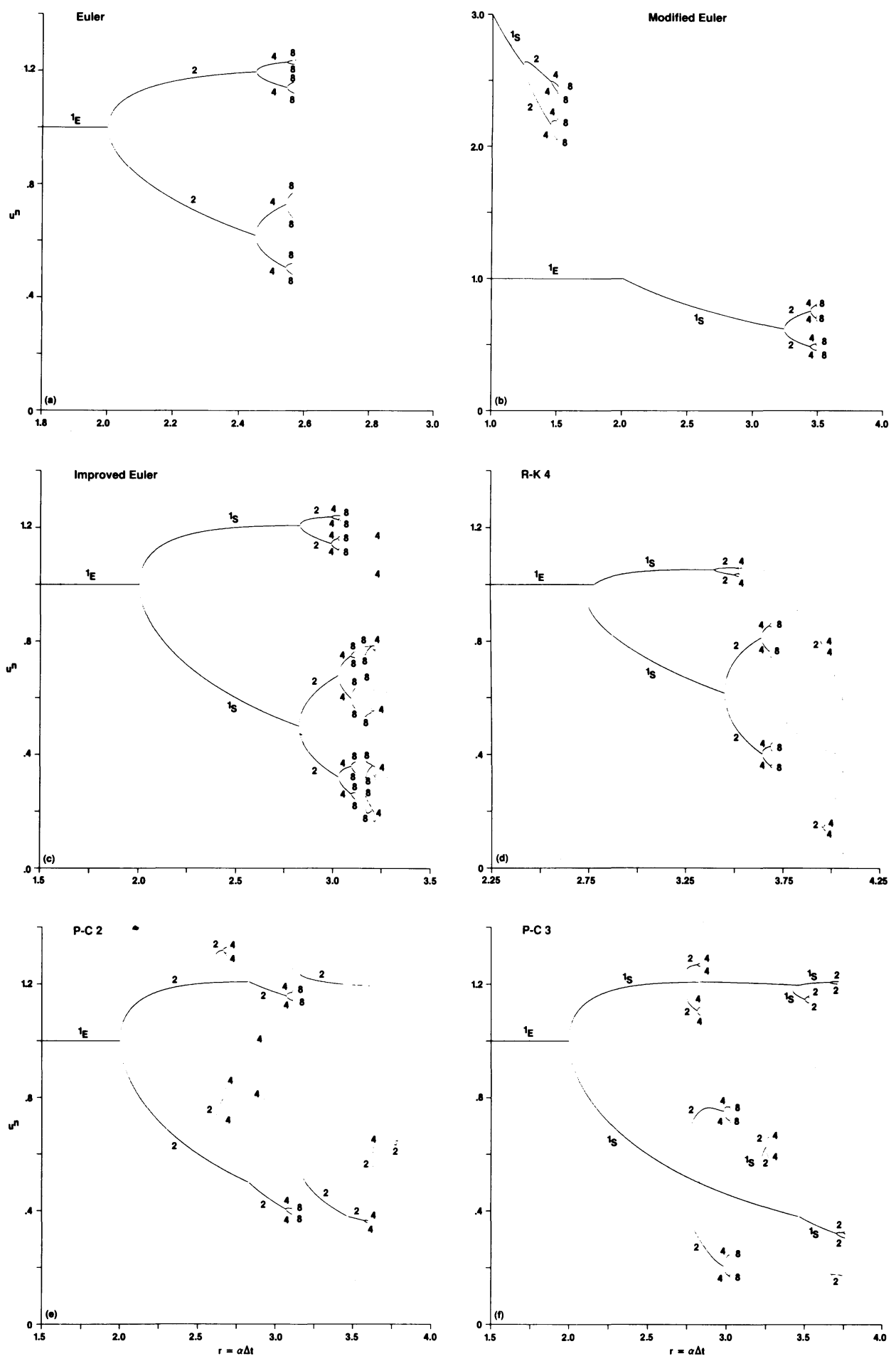
R-K 4 method,

$$
\begin{aligned}
u^{n+1} & =u^{n}+\frac{r}{6}\left(k_{1}+2 k_{2}+2 k_{3}+k_{4}\right) \\
k_{1} & =S^{n} \\
k_{2} & =S\left(u^{n}+\frac{r}{2} k_{1}\right) \\
k_{3} & =S\left(u^{n}+\frac{r}{2} k_{2}\right) \\
k_{4} & =S\left(u^{n}+r k_{3}\right) .
\end{aligned}
$$

Predictor-corrector method of order $m$,

$$
\begin{aligned}
u^{(0)} & =u^{n}+r S^{n} \\
u^{(k+1)} & =u^{n}+\frac{r}{2}\left[S^{n}+S^{(k)}\right], \quad k=0,1, \ldots, m-1 \\
u^{n+1} & =u^{n}+\frac{r}{2}\left[S^{n}+S^{(m-1)}\right] .
\end{aligned}
$$

Using the same procedures, one can obtain the fixed points for each of the above schemes (3.19)-(3.22). Figures 3.2b-3.2f show the stable fixed point diagrams of period $1,2,4$, and 8 for these five schemes for $S(u)=u(1-u)$. Some of the fixed points of lower period were obtained by closed form analytic soution and/or by a symbolic manipulator such as MAPLE [84] to check against the computed fixed point. The majority were computed numerically $[3,9]$. The stability of these fixed points was examined by checking the discretized form of the appropriate stability conditions. Again the axis is divided into 1000 equal intervals. The numeric labelling of the branches denotes their period; although some labels for period 4 and 8 are omitted due to the size of the labelling areas. The subscript $E$ on the main period one branch indicates the stable fixed point of the DE while the subscript $S$ indicates the stable spurious fixed points introduced by the numerical scheme. Spurious fixed points of period higher than one are obvious (since the ODEs under discussion only possess steady-state solutions) and are not labelled except for special cases. Note that these diagrams, which for the most part appear to consist of solid lines, actually consist of points, which are only apparent in areas with high gradients.

To contrast the results, similar stable fixed point diagrams were also computed for $S(u)=u(1-u)(b-u), 0<b<1$. See Fig. 3.3. The stable fixed point for the ODE

FIG. 3.3. Stable fixed points of periods $1,2,4,8$ for the ODE $d u / d t=\alpha u(1-u)(0.5-u)$. 

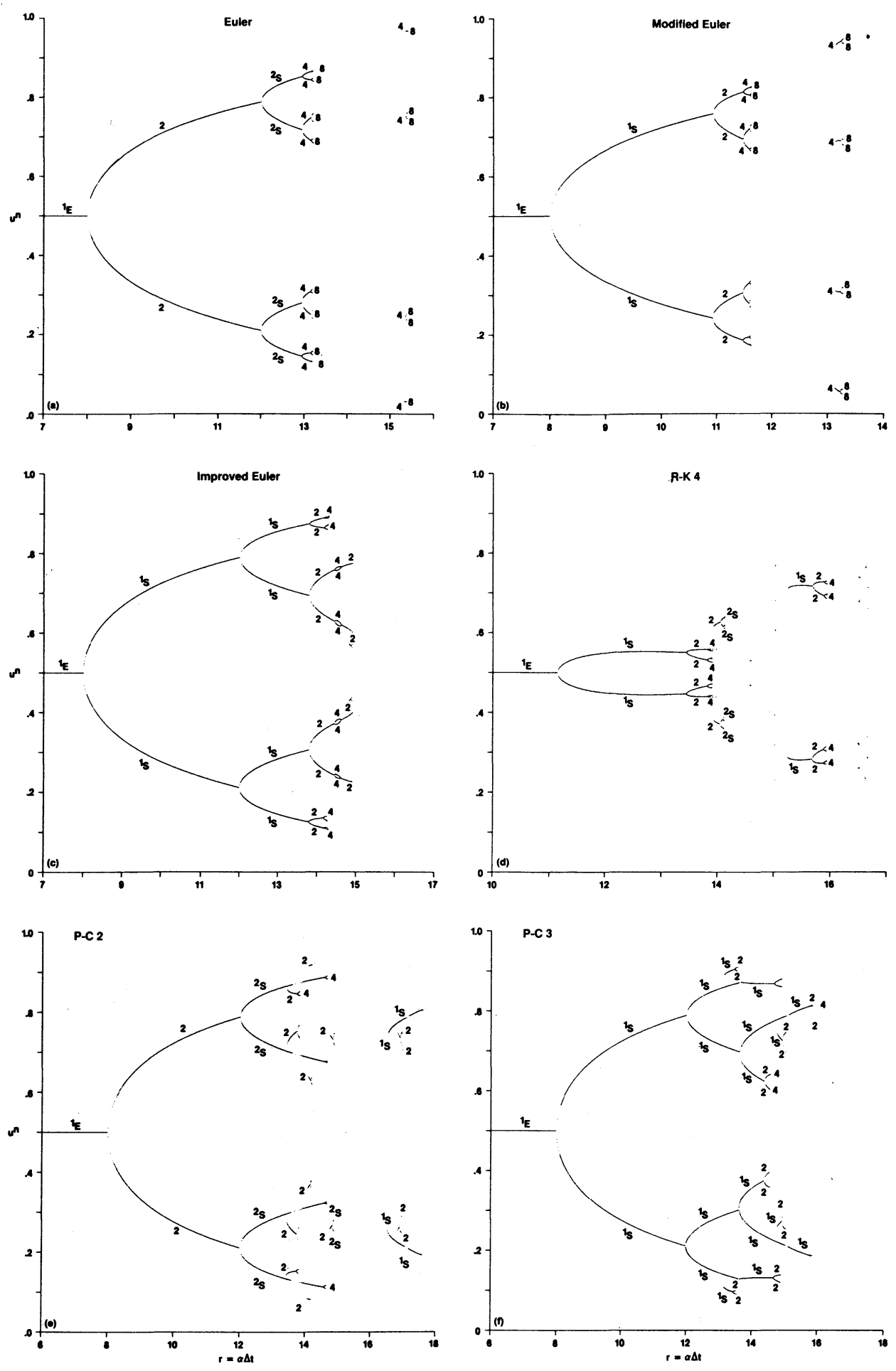
in this case is $u^{*}=b$ and the unstable ones are $u^{*}=0$ and $u^{*}=1$. For any $0<u^{0}<1$ and any $\alpha>0$, the solution will asymptotically approach the only stable asymptote of the ODE $u^{*}=b$.

In contrast to the asymptotic behavior of the ODEs, the maximum number of stable and unstable fixed points (real and complex) for each scheme varied from 4 to 16 for $S(u)=u(1-u)$ and 9 to 81 for $S(u)=u(1-u)(b-u)$, depending on the numerical method and the $r$ value. In other words, besides the exact fixed points, the extra fixed points are numerical artifacts of the underlying scheme. The domains of all of the fixed point diagrams are chosen so that they cover the most interesting part of the scheme and ODE combinations. Notice that spurious asymptotes might occur in other parts of the domain as well.

Aside from the striking difference in topography in the stable fixed point diagrams of the various methods and ODE combinations, all of these diagrams have one common feature: they all exhibit stable spurious fixed points, as well as stable spurious fixed points of period higher than one. In the majority of cases, these occur for values of $r$ above the linearized stability limit. But this is not always the case as in demonstrated in the modified Euler scheme applied to the logistic ODE and $d u / d t=\alpha u(1-u)(b-u), 0<b<0.5$, and the R-K 4 applied to the logistic ODE. For these two methods and ODE combinations, stable spurious fixed points occur below the linearized stability limit. In some of the instances, these spurious fixed points are outside the interval of the stable and unstable fixed points of the ODEs. Others not only lie below the linearized stability limit but also in the region between the fixed points of the DEs and so could be very easily achieved in practice. For example, in Fig. 3.2b, the modified Euler scheme for the logistic ODE, the linearized stability limit of period $1_{E}$ is $r=2$. But depending on the value of $r$, two stable fixed points of period 1 (one is spurious) can exist at the same time for $r$ between (approximately) the interval $(0.0,1.45)$. For the R-K 4 method applied to the logistic DE, one can see from Fig. 3.2d that spurious steady states which exist for $2.75<r<2.785$ are below the linearized stability limit of the $1_{E}$ branch. For the modified Euler method applied to $d u / d t=\alpha u(1-u)(b-u)$, it is interesting to see the changing behavior of stable spurious steady states as the stable fixed point $u^{*}=b$ is varied between 0 and 0.5. See Fig. 3.4 for details.

One might argue that for the ODEs that we are considering, it is trivial to check whether an asymptote is spurious or not. For example, if $\bar{u}$ is a spurious asymptote of period one, then $S(\bar{u}) \neq 0$. The main purpose of the illustration is to set the baseline dynamical behavior of the scheme so that one can use it wisely in other more complicated settings such as when nonlinear PDEs are encountered in which the exact solutions are not known. Under this situation, spurious asymptotes could be computed and mistaken for the correct steady-state solutions.

Note that for the modified Euler method, spurious fixed points of higher periods and chaotic attractors as well as spurious steady states occur below the linearized stability limit. Let $\Omega$ be the basin of attraction of the fixed point of the ODE and let $r^{*}$ be the corresponding linearized stability limit value of the scheme. Then there exists a portion of the basin $\Omega$ denoted by $\Omega^{c}$ in which $\Omega^{c} \subset \Omega$ and an interval of 

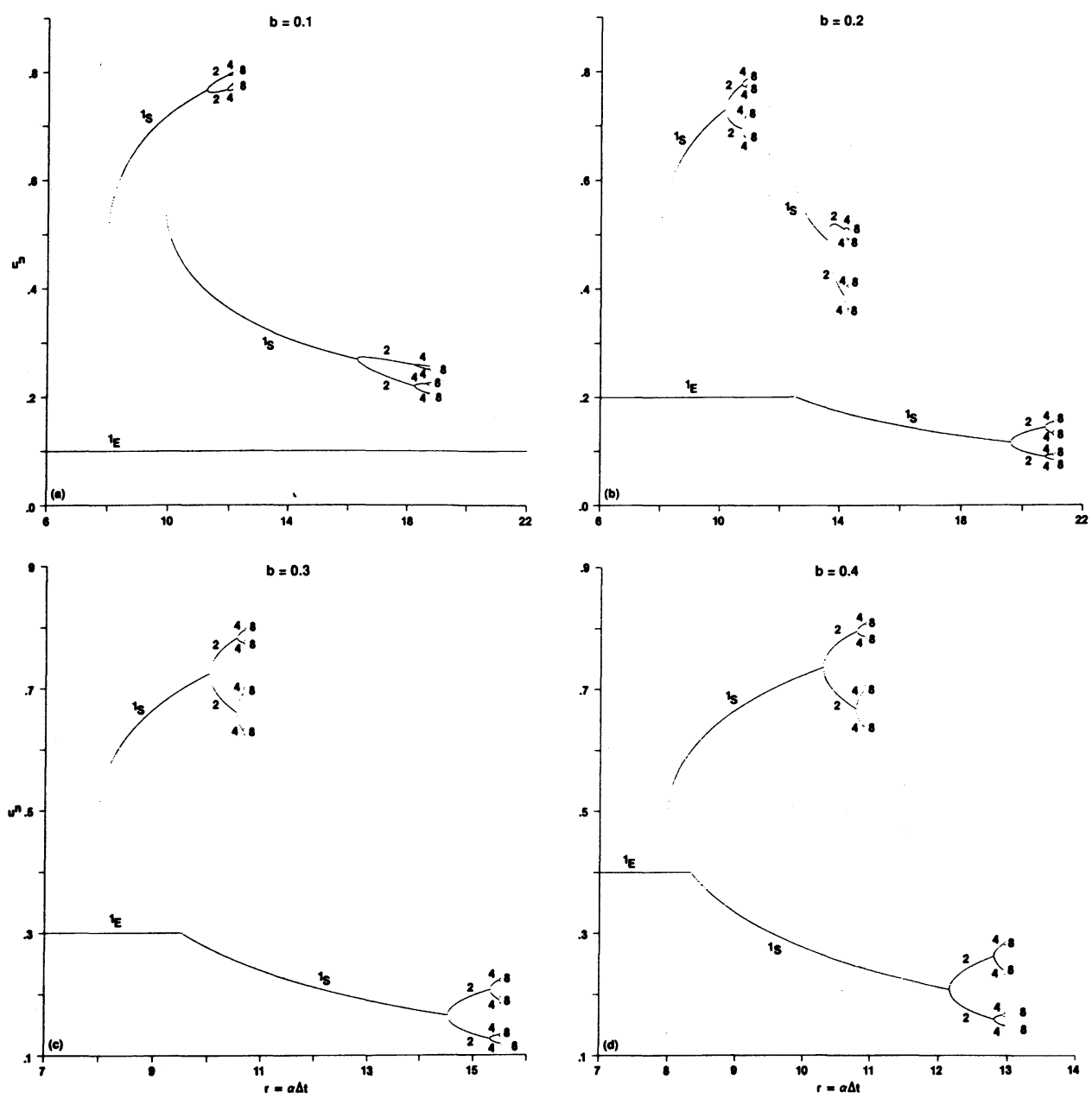

FIg. 3.4. Stable fixed points of periods $1,2,4,8$ of the modified Euler (R-K 2) scheme for the ODE $d u / d t=\alpha u(1-u)(b-u), b=0.1,0.2,0.3,0.4$.

$r$ within $\left[0, r^{*}\right)$ which actually belongs to the basin of attraction of the chaotic attractor of the discretized counterparts. There also exist some other $\Omega^{p} \subset \Omega$ and an interval of $r$ within $\left[0, r^{*}\right]$ and $p \geqslant 1$ an integer, which actually belongs to the basin of attraction of a stable asymptote of period $p$ of the corresponding discrete map. This leads to the issue of the dependence of solutions on initial data which will be the subject of the next subsection.

\subsection{Strong Dependence of Solutions on Initial Data}

For simple nonlinear ODEs that we are considering, the fixed point diagram is extremely useful for the understanding of the dynamics of the DEs and their dis- 
cretized counterparts. However, when fixed points of higher period and/or complex nonlinear DEs are sought, searching for the roots and testing for stability of highly complicated nonlinear algebraic equations can be expensive and might lead to inaccuracy.

Equally useful for the understanding the dynamics are the bifurcation diagram and basin of attraction of fixed points for both the DEs and the difference schemes. The bifurcation diagram for the one-dimensional discrete map displays the iterated solution $u^{n}$ vs $r$ after iterating the discrete map for a given number of iterations with a chosen initial condition (or multiple initial conditions) for each of the $r$ parameter values.

The term bifurcation is broadly used to describe significant qualitative changes that occur in the orbit structure of a dynamical system as the system parameters are varied. In general, bifurcation theory can be divided into two classes, local and global. Local bifurcation theory is concerned with the bifurcation of fixed points of nonlinear equations and discrete maps. Global bifurcation theory is concerned with phenomena away from the fixed points. It studies the interaction between different type of fixed points. A fixed point is structurally stable if nearby solutions have qualitatively the same dynamics. The linearized stability limit of a fixed point of a

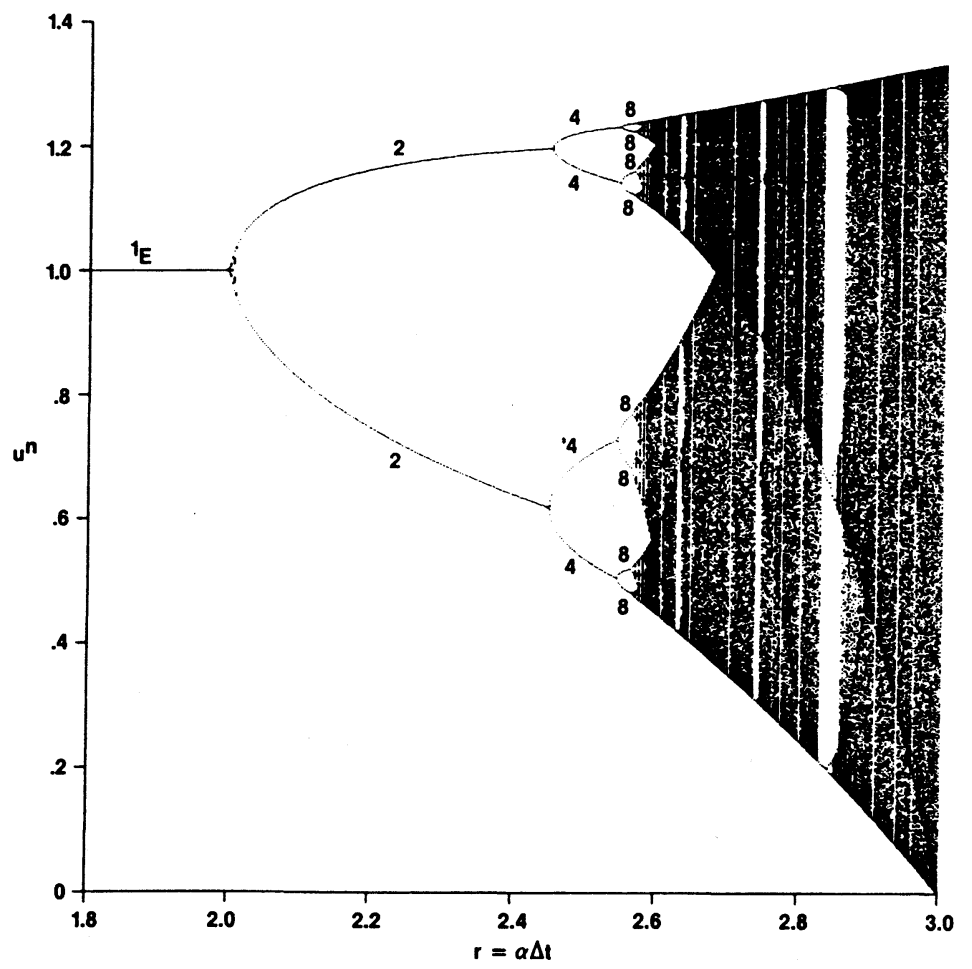

Fig. 3.5. Bifurcation diagram of the explicit Euler scheme for the logistic ODE $d u / d t=\alpha u(1-u)$. 
scheme is the same as the bifurcation point in the corresponding bifurcation diagram of the resulting discrete map.

For the numerical computations of the bifurcation diagrams with a given interval of $r$ and a chosen initial condition (or multiple initial conditions), the $r$ axis is divided into 500 equal spaces. In each of the computations, the discrete maps were iterated with 600 preiterations and the next 200 iterations were plotted for each of the $500 r$ values. The domains of the $r$ and $u^{n}$ axes are chosen to coincide with the stable fixed point diagrams shown previously. For our current interest, it is not necessary to distinguish the difference between a stable fixed point of period 200 and a chaotic attractor.

Figure 3.5 shows the bifurcation diagram of the Euler scheme applied to the
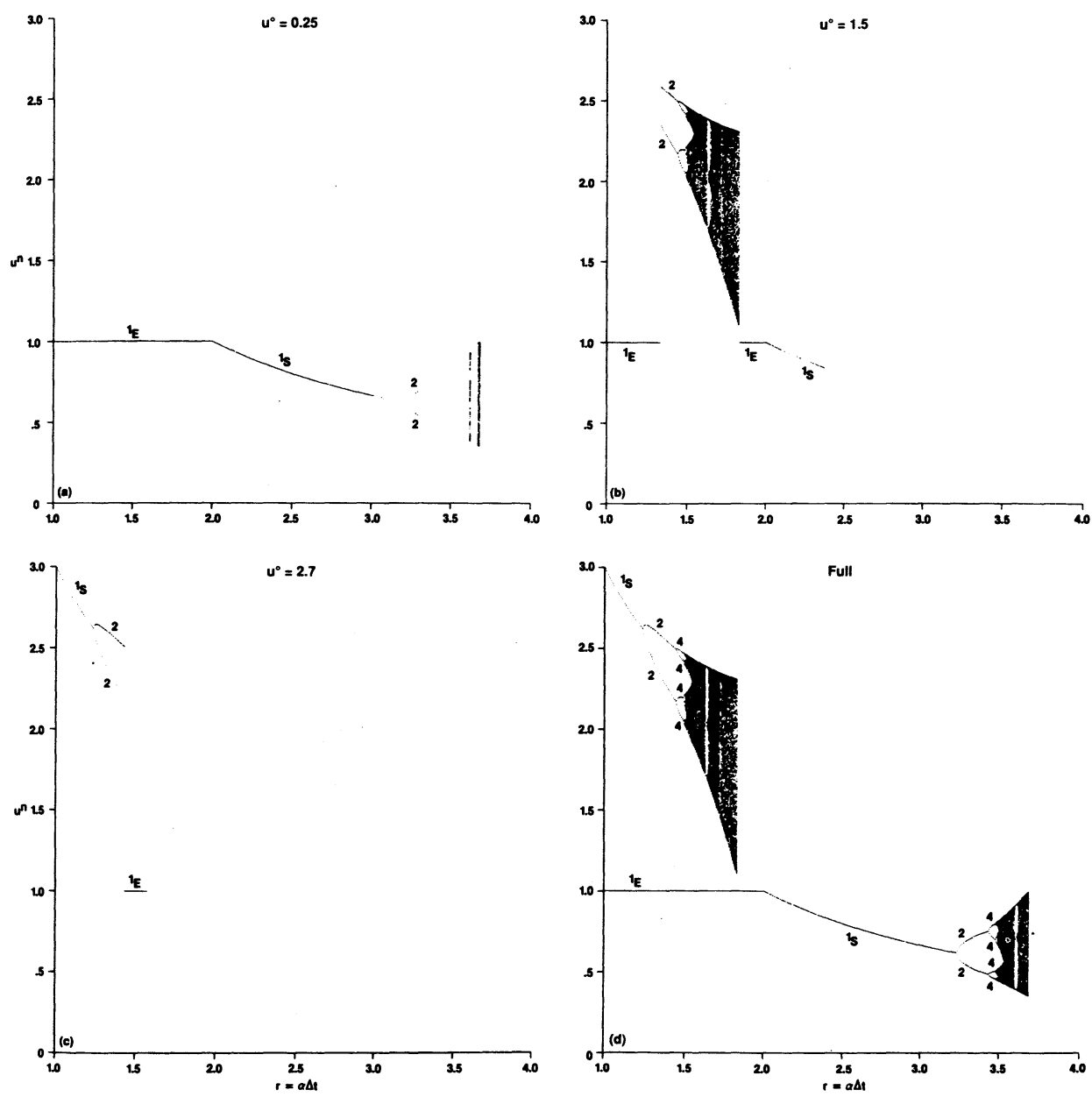

Fig. 3.6. Bifurcation diagrams of the modified Euler (R-K 2) scheme for the logistic ODE $d u / d t=$ $\alpha u(1-u)$. 
logistic DE with an initial condition $u^{0}>0$. It is of interest to know that in this case the bifurcation diagram looks practically the same for any $u^{0}>0$. This is due to the fact that no spurious fixed points or spurious asymptotes of low period exist for $r<2.627$. Comparing the bifurcation diagram with Fig. 3.2a, one can see that if we had computed all of the fixed points of period up to 200 for Fig. 3.2a, the resulting fixed point diagram would look the same as the corresponding bifurcation diagram (assuming 800 iterations of the logistic map are sufficient to obtain the converged stable asymptotes of period up to 200 and a proper set of initial data are chosen to cover the basins of all of the periods in question). The numeric labellings of the
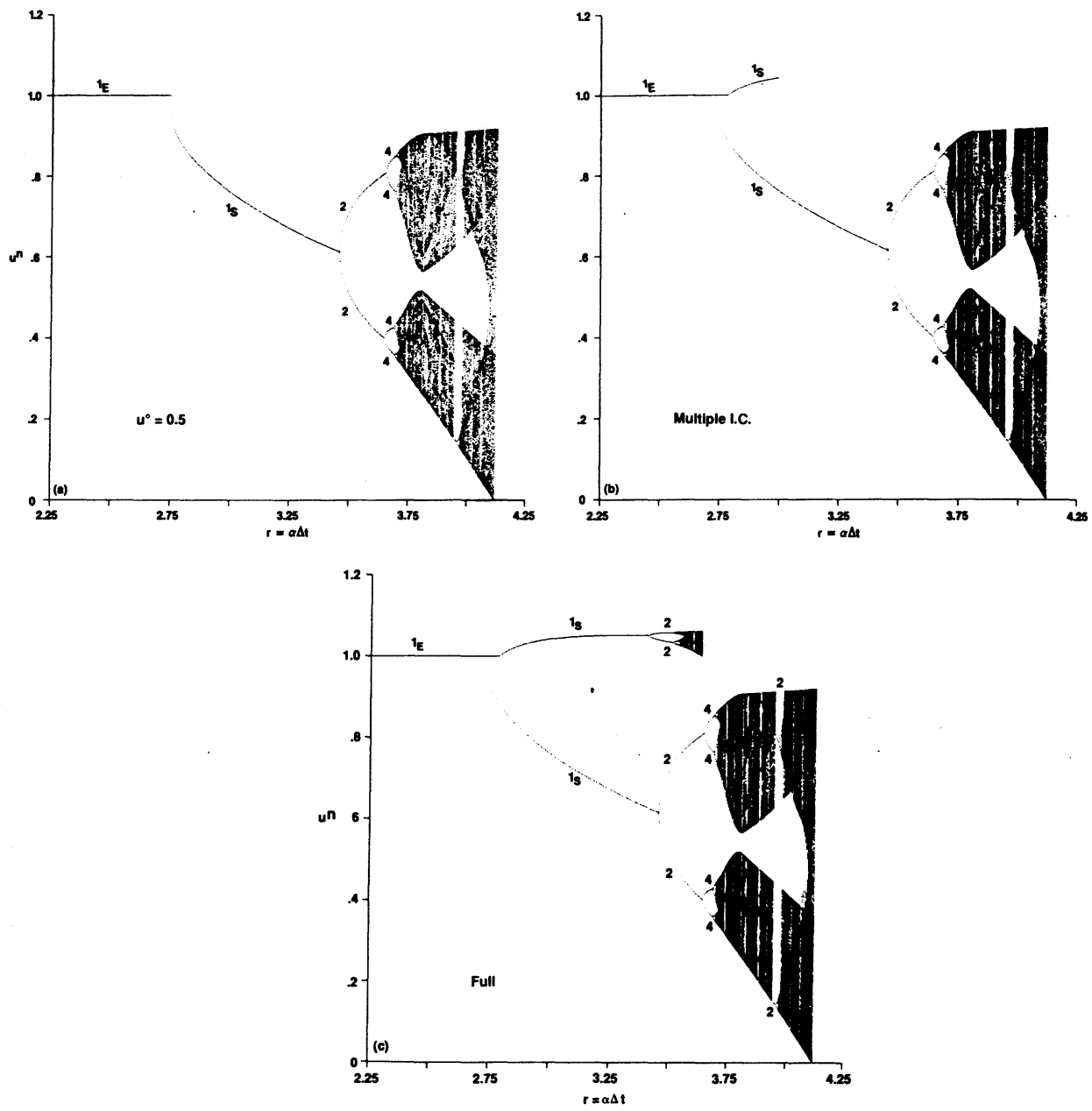

Fig. 3.7. Bifurcation diagrams of the Runge-Kutta fourth-order (R-K 4) scheme for the logistic ODE $d u / d t=\alpha u(1-u)$. 
branches in the bifurcation diagram denote their period, with only the essential ones labelled for identification purposes.

The noise appearing on the $1_{E}$ branch near the bifurcation point $r=2$ of the linearized stability limit of the fixed point $u^{*}=1$ indicates that 800 iterations of the logistic map is not sufficient to obtained the converged stable asymptotes. This phenomenon is common to other bifurcation points of higher periods as well as the rest of the bifurcation diagrams (Figs. 3.6-3.12) to be discussed shortly.

In order to interpret the bifurcation diagram for other ODE and scheme combinations, some knowledge of the fixed point diagram is necessary, at least for the
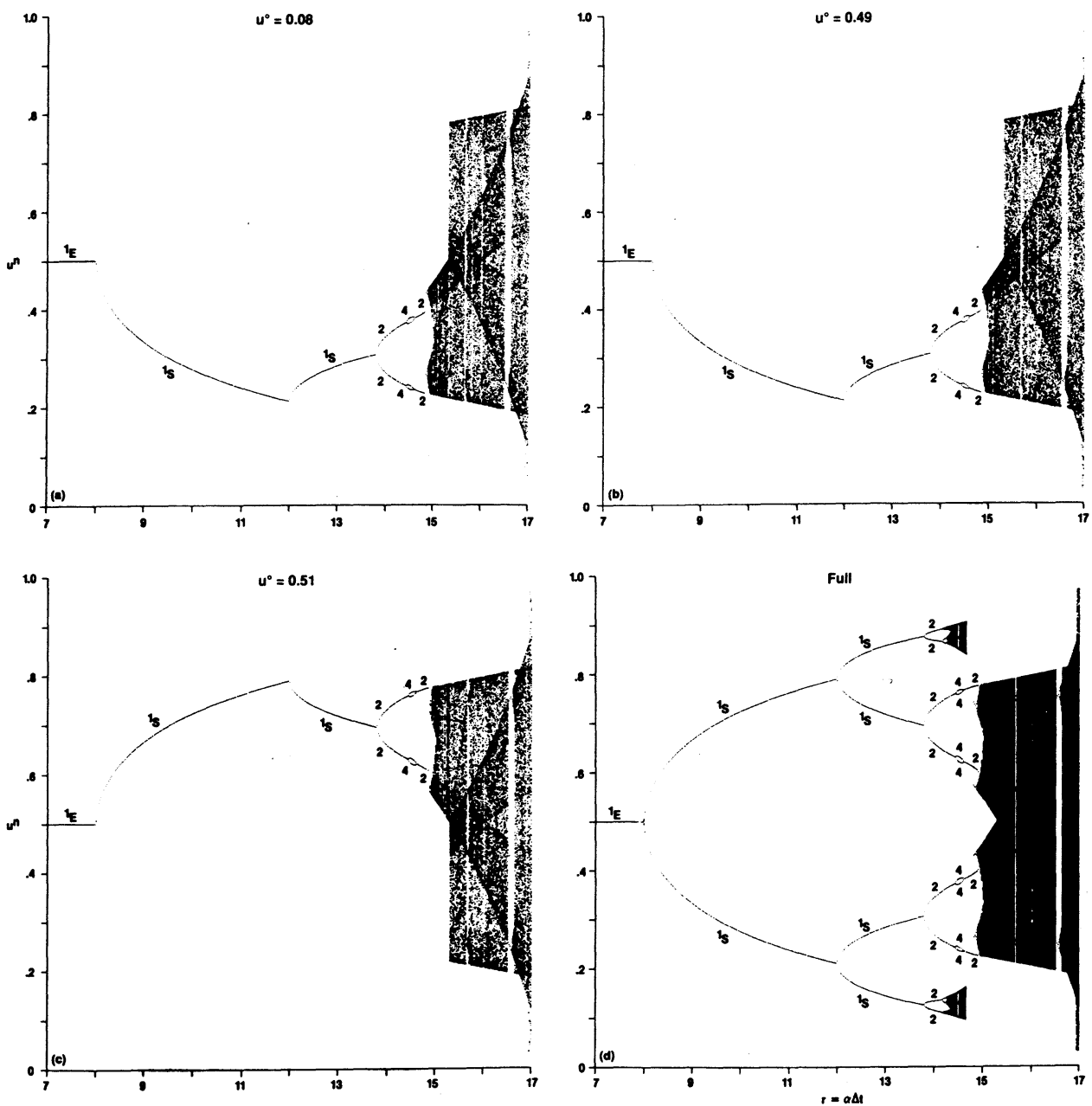

FIG. 3.8. Bifurcation diagrams of the improved Euler (R-K 2) scheme for the ODE $d u / d t=$ $\alpha u(1-u)(0.5-u)$ for four different sets of initial input data. 

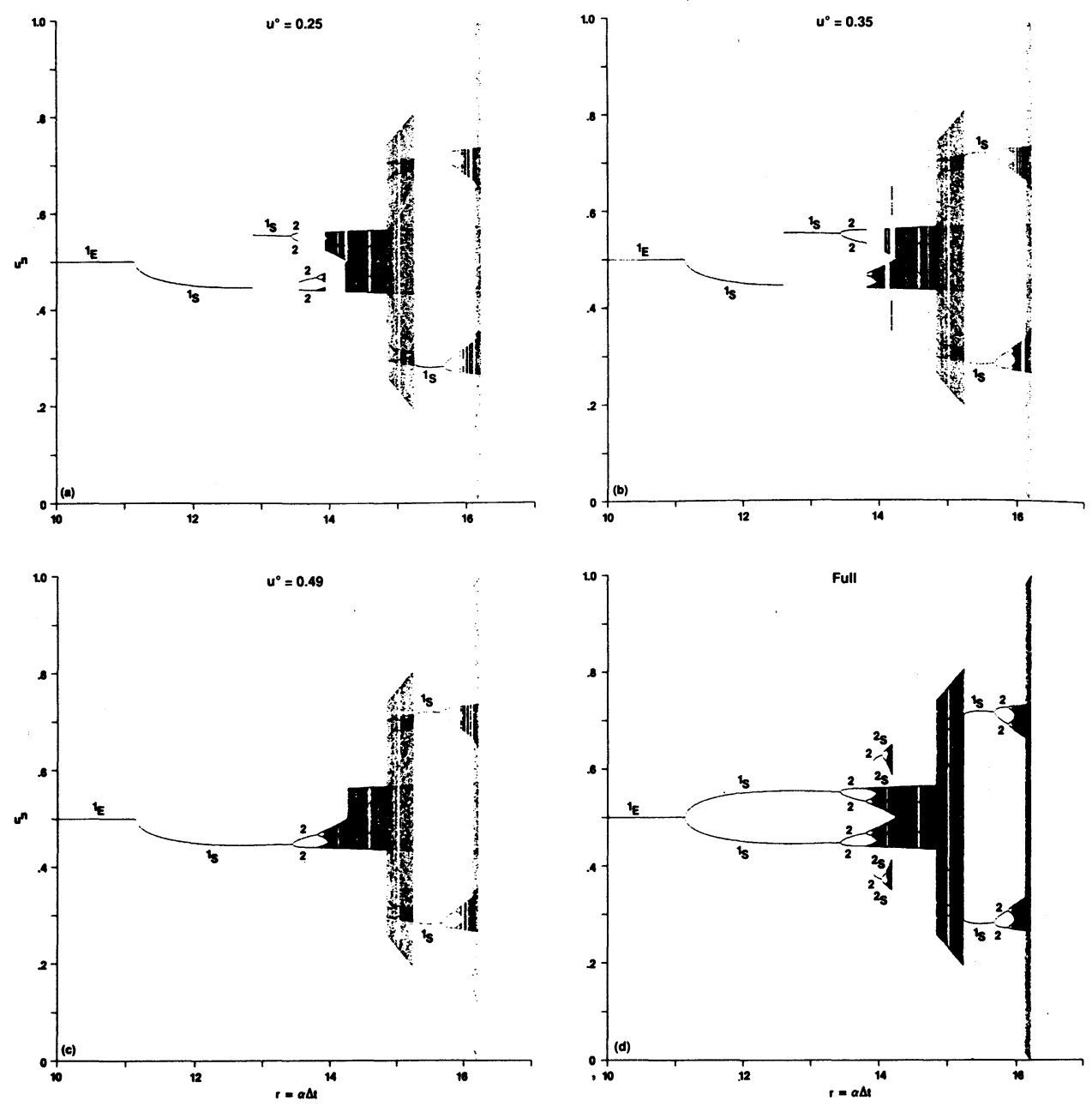

FIG. 3.9. Bifurcation diagrams of the Runge-Kutta fourth-order (R-K 4) scheme for the ODE $d u / d t=\alpha u(1-u)(0.5-u)$ for four different sets of initial input data.

lower order periods. Otherwise, one cannot identify the exact periodicity of the asymptotes easily. As can be seen later, a "full" bifurcation diagram cannot be obtained efficiently without the aid of the stable and unstable fixed point diagram for schemes that exhibit spurious fixed points of any period, especially lower periods. In most cases, the unstable asymptotes divide the domain into the proper basins of attraction for the stable asymptotes (spurious or otherwise), and at least one initial data point is used from each of the basins of attraction before a full bifurcation diagram can be obtained.

FIG. 3.10. "Full" bifurcation diagrams for the logistic ODE $d u / d t=\alpha u(1-u)$. 

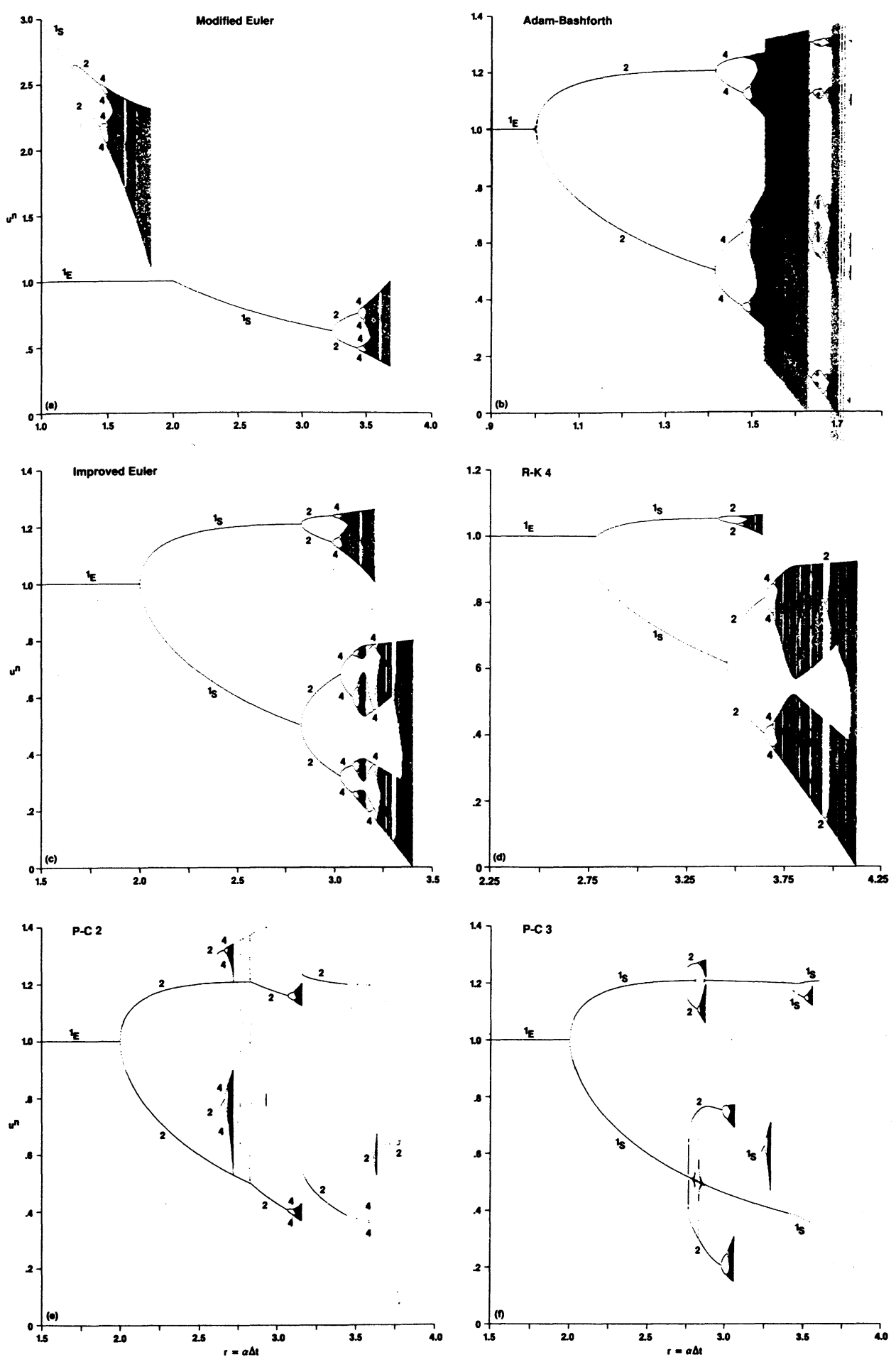
In all of the fixed point diagrams 3.2-3.4, the bifurcation phenomena can be divided into three kinds. For the first kind, the paths (spurious or otherwise) resemble period doubling bifurcations (flip bifurcation) [3-6] similar to the logistic map. See Figs. 3.2a, 3.2e, 3.3a, and 3.3e for examples. The second kind occurs, most often, at the main branch $1_{E}$, with the spurious paths branching from the correct fixed point as it reaches the linearized stability limit, and quite often even bifurcating more than once (pitchfork bifurcation or supercritical bifurcation [85, 8]), as $r$ increases still further before the onset of period doubling bifurcations. See Figs. 3.2c, 3.2f, 3.3b-3.3d, and 3.3f for examples. The third kind again occurs most often at the main branch $1_{E}$. The spurious paths near the linearized stability limit of $1_{E}$ experience a transcritical bifurcation $[4,8,10,85]$. See Figs. 3.2b, 3.2d, 3.2f, and 3.4 for examples. Notice that the occurrence of transcritical and supercritical bifurcations is not limited to the main branch $1_{E}$. See Figs. 3.3d-3.3f and 3.4 for examples. The other commonly occurring bifurcation phenomenon is the subcritical bifurcation which was not observed in our two chosen $S(u)$ functions. With a slight change in the form of our cubic function $S(u)$, a subcritical bifurcation can be achieved $[85,4,8,10]$. The consequence of the latter three bifurcation behaviors is that bifurcation diagrams calculated from a single initial condition $u^{0}$ will appear to have missing sections of spurious branches, or even seem to jump between branches. This is entirely due to the existence of spurious asymptotes of some period or more than one period, and its dependence on the initial data. This occurs even for the Euler scheme as depicted in Fig. 3.3a. See Section 3.4 for further discussion of these four types of bifurcation phenomena.

Figures 3.6a-3.6c show the bifurcation diagram by the modified Euler method for the logistic ODE with three different starting initial conditions. In contrast to the explicit Euler method as shown in Fig. 3.5 none of these diagrams look alike. One can see the influence and the strong dependence of the asymptotic solutions on the initial data. Thus in a situation where there is no prior information about the exact steady-state solution and where a time-dependent approach is used to obtain the steady-state numerical solution when initial data are not known, stable spurious steady-state could be computed and mistaken for the correct steady-state solution. Figure 3.6d shows the corresponding "full" bifurcation diagram, their earlier stages resembling the fixed point diagram 3.2b. Figure 3.7 illustrates similar bifurcation behavior for the corresponding R-K 4 method. Figure $3.7 \mathrm{~b}$ serves as an example to illustrate that the effect of overplotting a number of initial data, but not the appropriate ones, would not be sufficient to cover all of the essential spurious branches. In Fig. 3.7b, I.C. stands for initial condition. Figures 3.8-3.9 show a similar illustration for $S(u)=u(1-u)(0.5-u)$ by the improved Euler and the $\mathrm{R}-\mathrm{K} 4$ methods. The strong dependence of solutions on initial data is evident from the various examples in which this type of behavior is very common for genuinely nonlinear problems.

FIG. 3.11. "Full" bifurcation diagrams for the ODE $d u / d t=\alpha u(1-u)(0.5-u)$. 

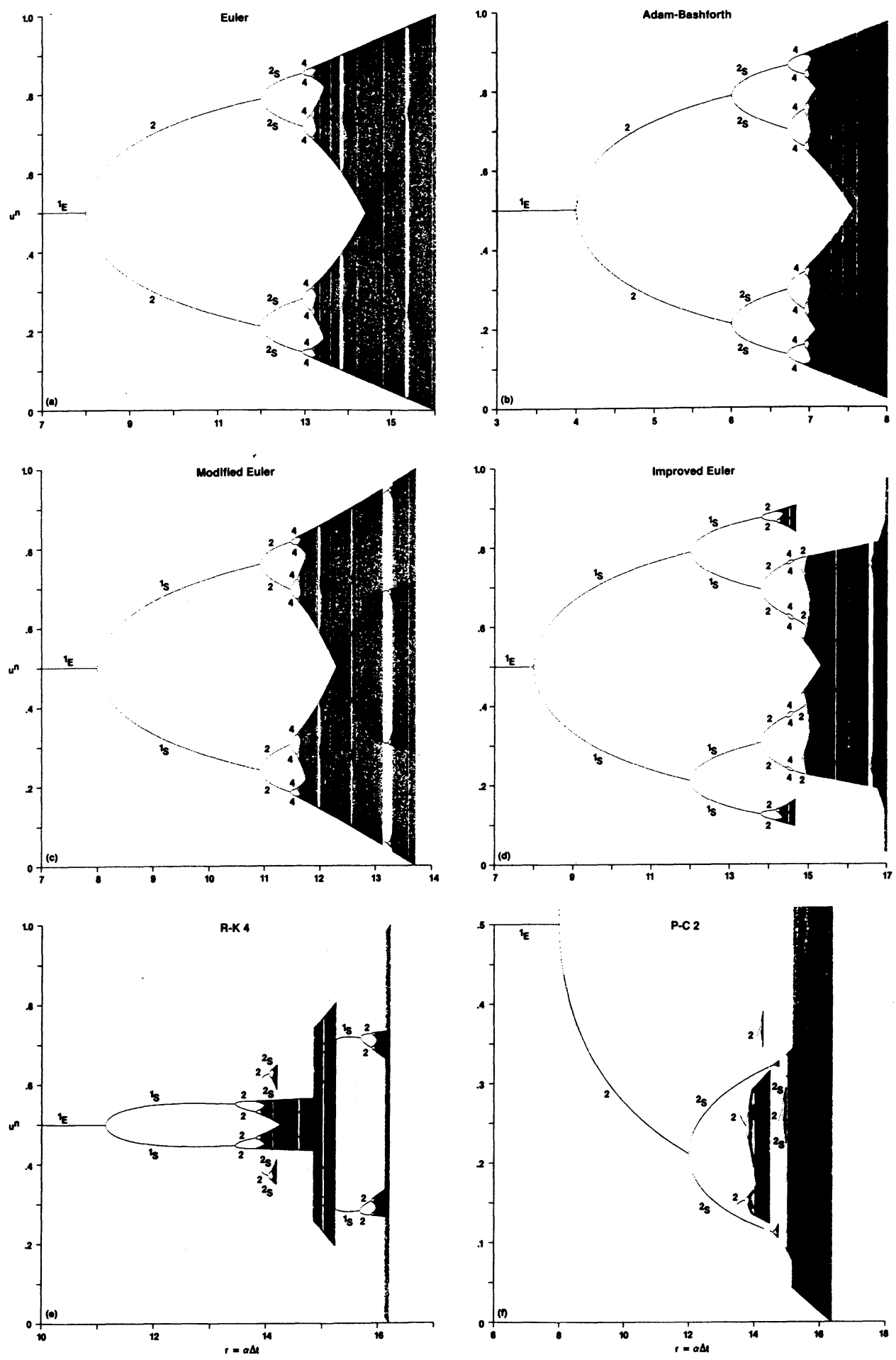
In order to compute a "full" bifurcation diagram, we must overplot a number of diagrams obtained by the guide of the stable and unstable fixed point diagram as an appropriate set of starting initial data. In the case where the fixed point diagrams are extremely difficult to compute, a brute force method of simply dividing the domain of interest of the $u^{n}$ axis into equal increments and using these $u^{n}$ values as initial data is employed. The "full" bifurcation diagram is obtained by simply overplotting all of these individual diagrams on one.

For completeness, Figs. 3.10-3.12 show the "full" bifurcation diagrams for the corresponding fixed point diagrams shown previously. Figures 3.13-3.15 shows the
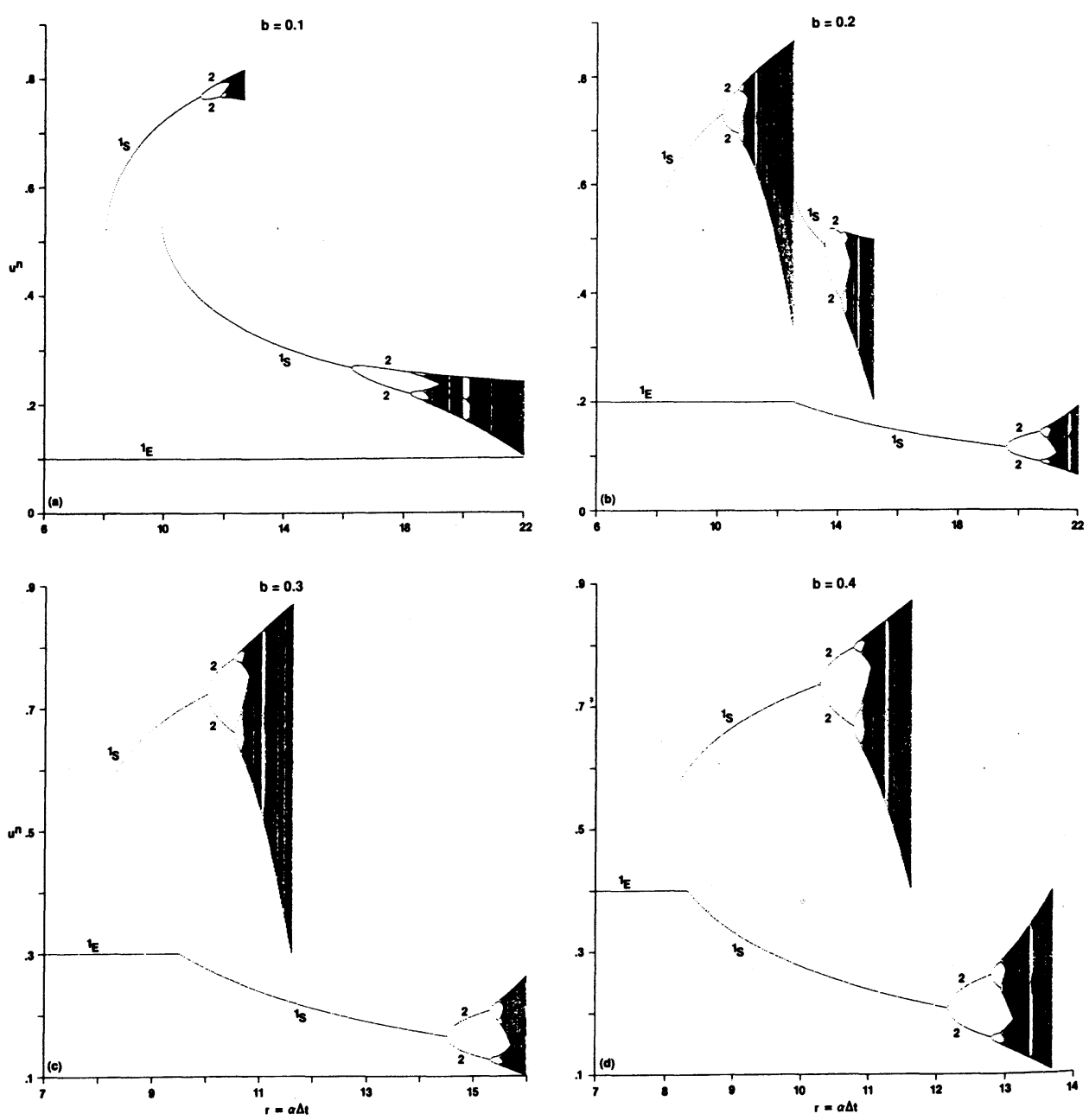

FIG. 3.12. "Full" bifurcation diagrams of the modified Euler (R-K 2) scheme for the ODE $d u / d t=$ $\alpha u(1-u)(b-u), b=0.1,0.2,0.3,0.4$. 
stable and unstable fixed point diagrams used as a guide to pick the appropriate initial data to obtain the full bifurcation diagrams 3.10-3.12. The number of initial data points used in each of the "full" bifurcation diagrams ranges from 4 to as high as 20. Notice that the exact values of the initial data are immaterial as long as these values cover all of the basins of attraction of the essential lower order periods. That is, at least one initial data point is used from each of the basins of the essential lower order periods (using the stable and unstable fixed point diagrams as a guide). See Section 3.4 for additional details and their connection with the basins of attraction. Here, we use the term "full bifurcation diagram" to mean "bifurcation diagram with sufficient initial data to cover the essential lower order periods." No attempt has been made to compute the true full bifurcation diagram since this is very costly and involves a complete picture of the basins of attraction for the domain of interest in question.

\subsection{Classification of ODE Solvers \\ (According to the Existence of Spurious Fixed Points)}

In Ref. [27], Iserles studied the stability of ODE solvers for nonlinear autonomous ODE via the dynamical approach. He proved that LMM [81-83] that give bounded values at infinity always produce correct asymptotic behavior. However, this is not the case with Runge-Kutta methods and some predictorcorrector methods. He demonstrated that the Runge-Kutta and predictor methods may lead to false asymptotes, but did not discuss the possibility of these spurious asymptotes existing below the linearized stability limit.

For implicit LMM, he assumed the resulting nonlinear algebraic equations are solved exactly. He also showed the influence of nonlinear algebraic solvers on the size of stability regions for implicit LMM. His conclusion was that the standard nonlinear algebraic solver-the modified Newton-Raphson method (for the trapezoidal rule)

$$
u_{n+1}^{(k+1)}=u_{n+1}^{(k)}-\frac{u_{n+1}^{(k)}-u_{n}-(r / 2)\left[S\left(u_{n}\right)+S\left(u_{n+1}^{(k)}\right)\right]}{1-(r / 2) S_{u}\left(u_{n}\right)}
$$

can drastically degrade the region of stability limit as compared to the NewtonRaphson method

$$
u_{n+1}^{(k+1)}=u_{n+1}^{(k)}-\frac{u_{n+1}^{(k)}-u_{n}-(r / 2)\left[S\left(u_{n}\right)+S\left(u_{n+1}^{(k)}\right)\right]}{1-(r / 2) S_{u}\left(u_{n+1}^{(k)}\right)} .
$$

On the other hand, the direct iteration method

$$
u_{n+1}^{(k+1)}=u_{n}+\frac{r}{2}\left[S\left(u_{n}+S\left(u_{n+1}^{(k)}\right)\right]\right.
$$



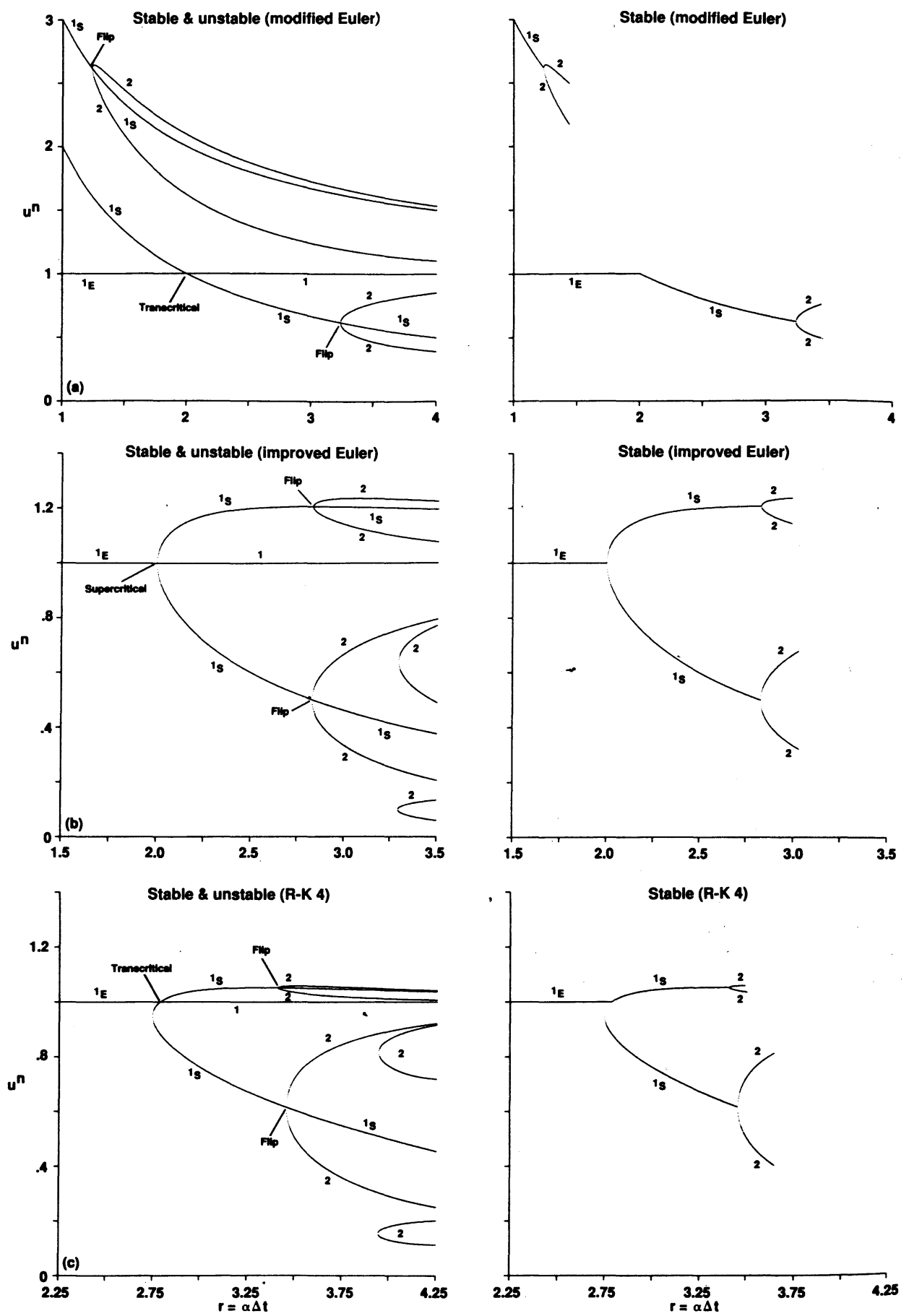

Fig. 3.13. Stable and unstable fixed points of periods 1,2 for the logistic ODE $d u / d t=\alpha u(1-u)$. 

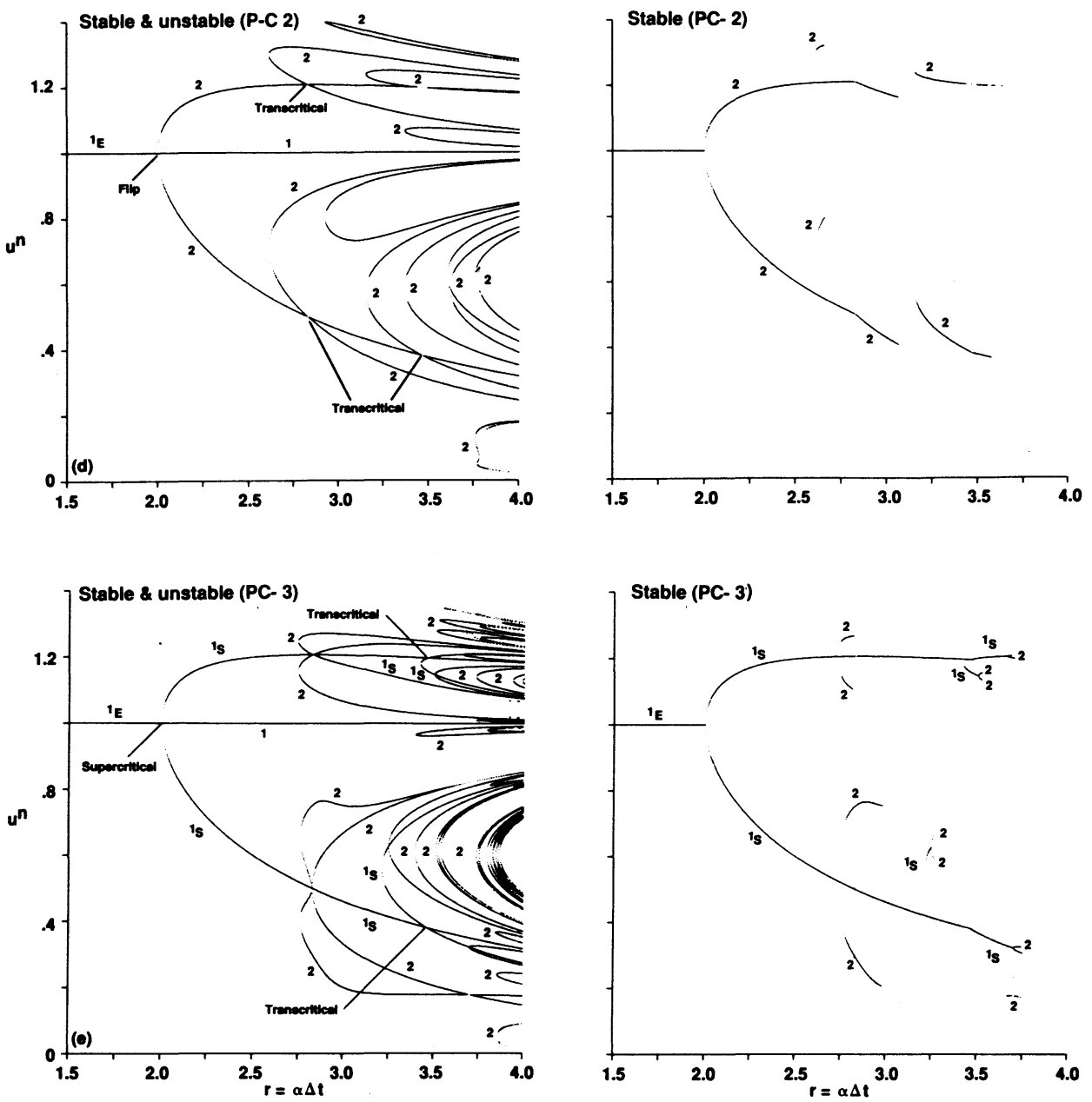

FIG. 3.13-Continued

converges only if the step size is of the same order of magnitude as that required for an explicit method. Thus the advantage of using an implicit method (3.25) to enhance stability is lost. Here for clarity of notation, when iteration procedures are involved, $u_{n}$ is used in place of $u^{n}$ of the previous section.

The implications of behavior detailed in Iserles' work [27] range far beyond pure ODEs. For most CFD applications, the use of implicit time discretization to "time" march the solution to steady state is very common. The resulting nonlinear algebraic systems are solved by either noniterative linearization $[86,2]$ or by some kind of iterative or relaxation procedure. Very often, applied computational fluid dynamicists experience a non-convergent solution where the residual will decrease 

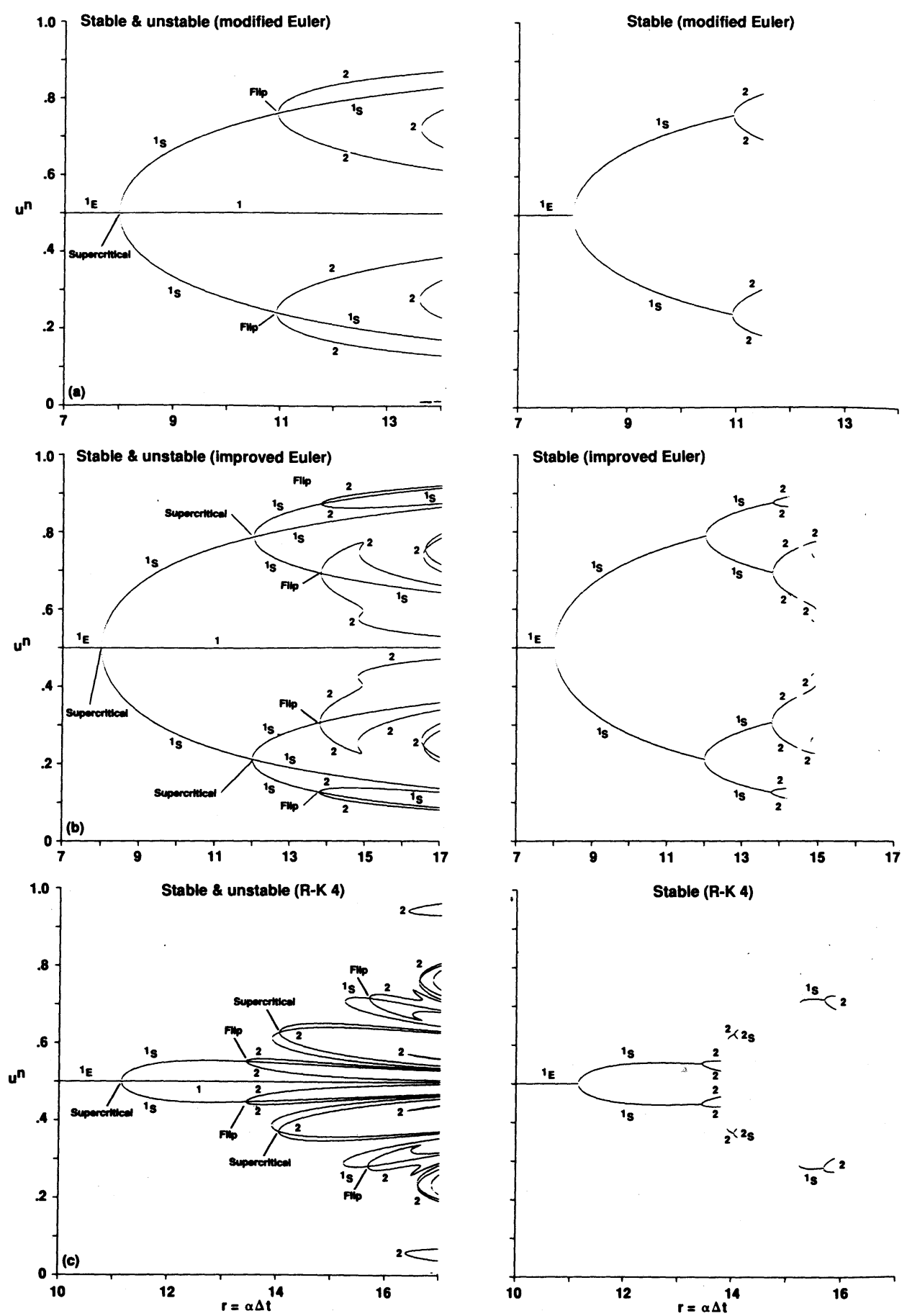

FIG. 3.14. Stable and unstable fixed points of periods 1,2 for the ODE $d u / d t=\alpha u(1-u)(0.5-u)$. 

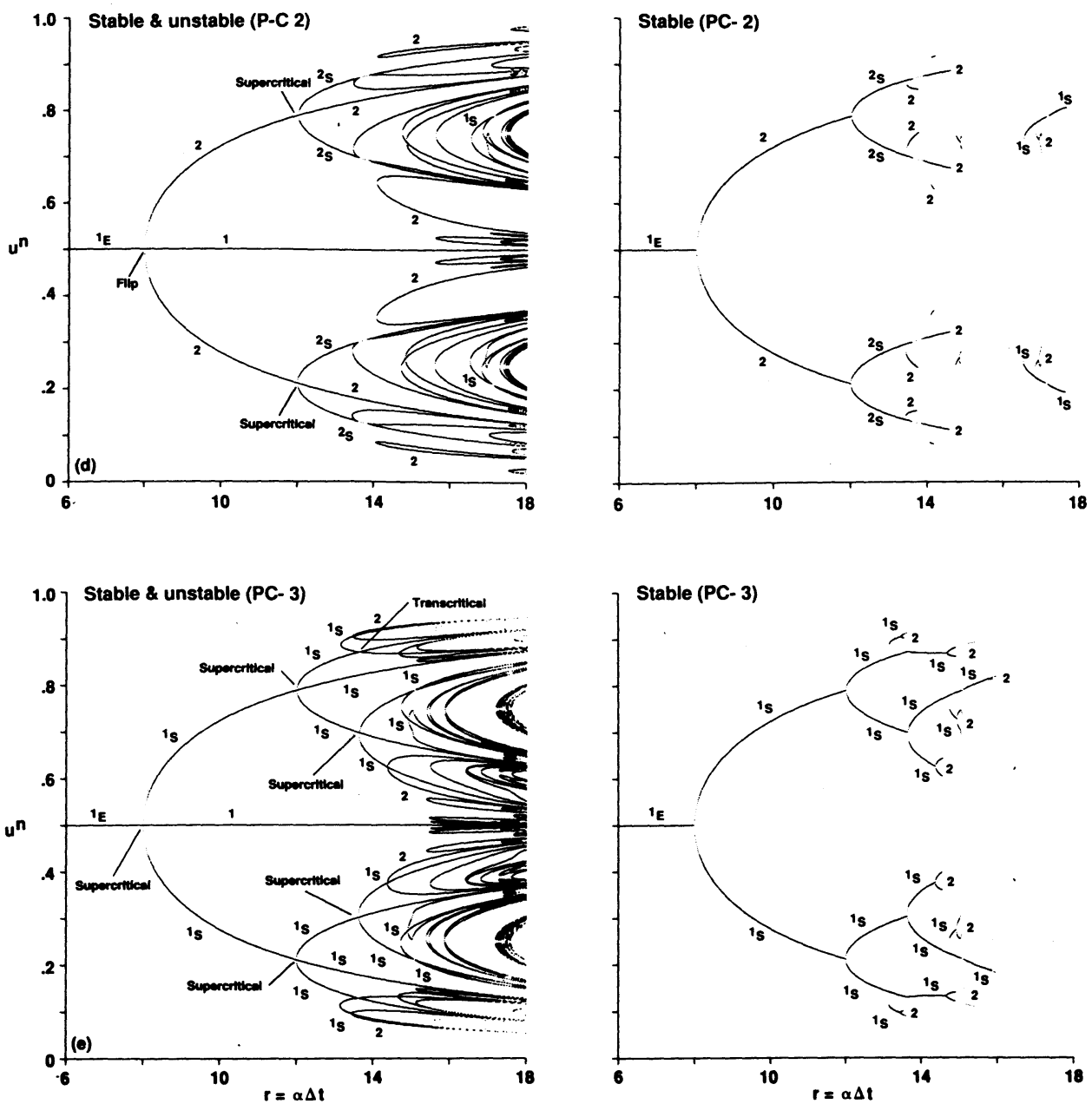

FIG. 3.14-Continued

only so far before reaching a plateau with a time step larger than the explicit method. The behavior observed in Iserles' work could explain the degradation in the stability of the implicit scheme in practice. Indeed, even though the mechanisms involved are far more complicated than those studied here, elements such as spatial discretization dynamical behavior and nonlinear coupling effect for systems, could well be an explanation.

More recently, Iserles and Sanz-Serna [28] established conditions for using a variable step size analysis to avoid spurious fixed points in a class of Runge-Kutta methods. A more up-to-date unified discussion on LMM, Runge-Kutta methods, and predictor-corrector methods has appeared in Refs. [29, 30]. 

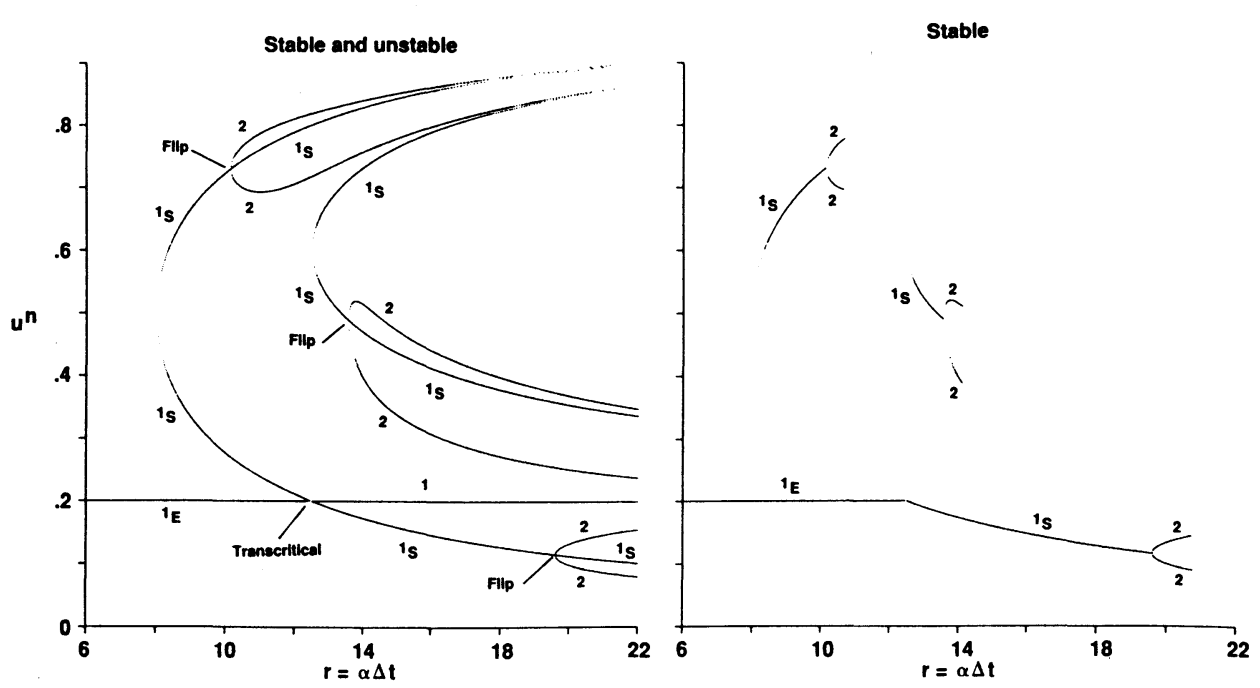

Fig. 3.15. Stable and unstable fixed points of periods 1,2 of the modified Euler (R-K 2$)$ scheme for the ODE $d u / d t=\alpha u(1-u)(0.2-u)$.

Looking at the problem from another perspective, it is very useful to find the cause of the existence of spurious asymptotes by looking at the form and properties of the resulting discrete maps, regardless of the methods. We have the following two observations:

(1) Assume that the only parameter that was introduced by a numerical method is $\Delta t$. Then from Iserles' results and our current investigation, one necessary condition for the existence of spurious steady states of ODE solvers for (3.1) is the introduction of nonlinearity in the parameter space $\Delta t$. This is evident from our examples and general analysis. For example, if $\Delta t$ (or $r$ ) is linear in (3.3), then (3.3) can be written as

$$
u^{n+1}=u^{n}+c r S\left(u^{n}\right), \quad c \text { a constant of the scheme. }
$$

Therefore, any fixed point of (3.3) is a fixed point of (3.1). Without loss of generality, a similar proof applies to the resulting difference operator $D$ from a $p$ time level LMM scheme.

(2) One can classify the types of spurious steady states in the form of bifurcation theory near a bifurcation point or a bifurcation limit point. Figures 3.16 and 3.17 show the definition of the various types of branching points and the stability of solutions in the neighborhood of branch points. In other words, for bifurcation of the same period, the classification is according to the onset of spurious asymptotes of subcritical, supercritical, or transcritical bifurcations. See Fig. 3.18 for the definition of the three types of phenomena. We refer the reader to Refs. $[4,6,8,10]$ for details of the various definitions. 


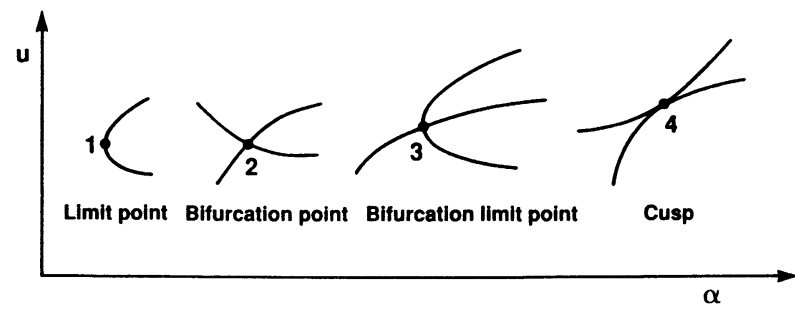

FIG. 3.16. Types of branching points.

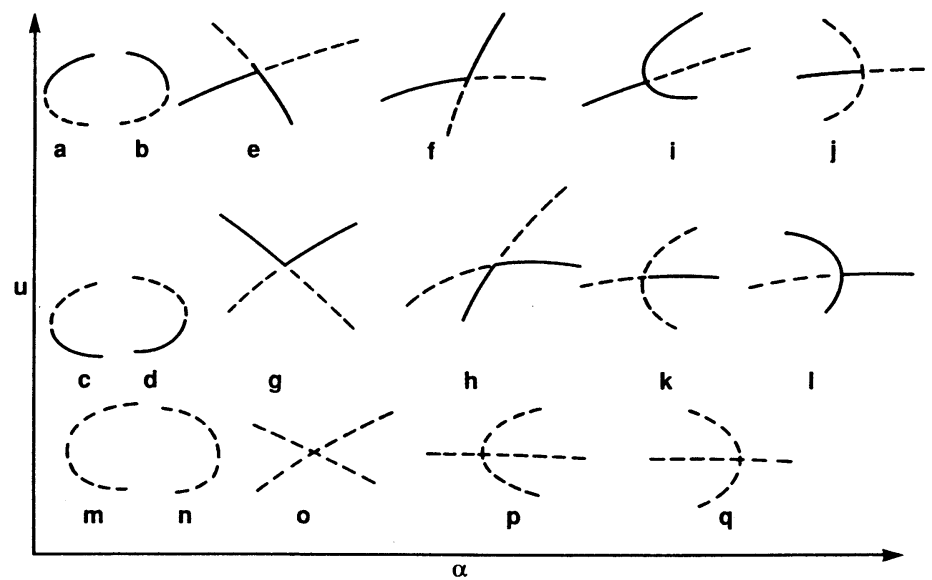

FIG. 3.17. Stability of soutions in the neighborhood of branch points, one-dimensional case. stable, --- unstable, a, b, c, d: limit (regular turning) point; e, f, g, h: bifurcation (double) points; $\mathrm{i}, \mathrm{j}, \mathrm{k}, \mathrm{l}$ : bifurcation-limit (singular turning) points; $\mathrm{m}, \mathrm{n}, \mathrm{o}, \mathrm{p}$, q: additional possible cases when the dimension of $u$ is greater than one (this figure is taken from Ref. [8]).

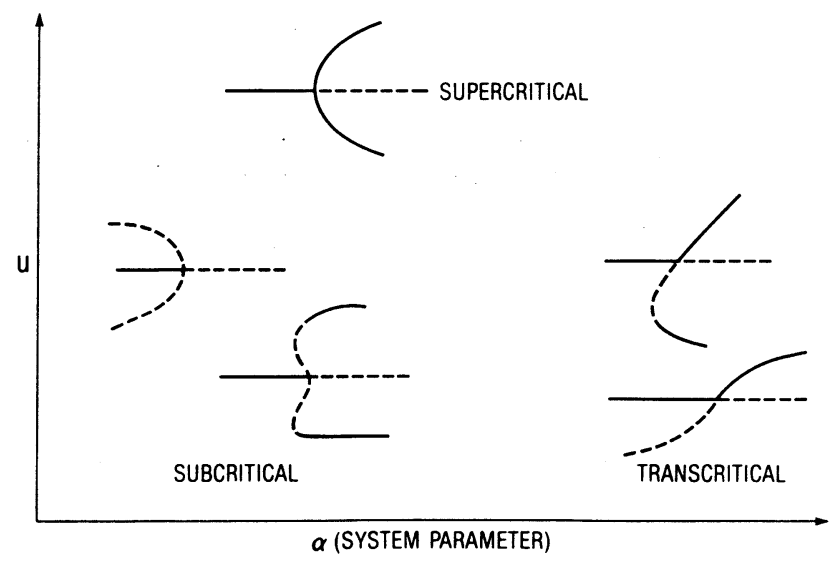

FIG. 3.18. Stability of steady-state solutions arising through three types of bifurcation phenomena stable, --- unstable). 

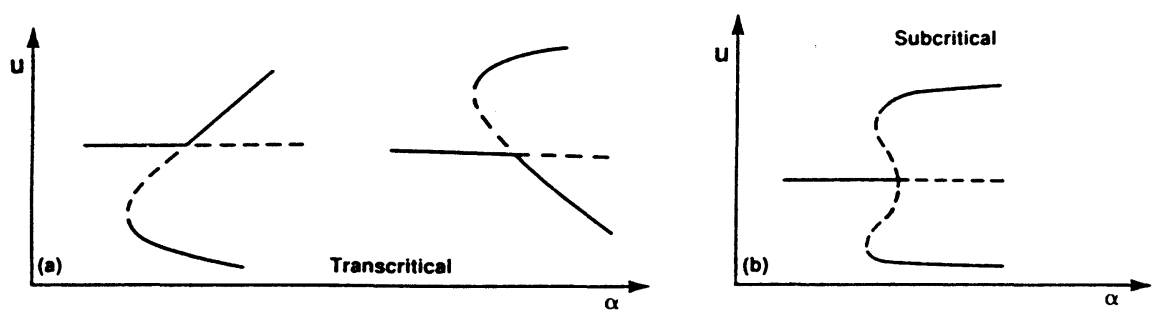

FIG. 3.19. Spurious fixed points arising from transcritical and subcritical bifurcations.

Assume an ODE solver introduces nonlinearity in the parameter space $\Delta t$ for (3.1). We hypothesize that a necessary and sufficient condition for the occurrence of spurious steady states below the linearized stability limit on the main branch $1_{E}$ (stable fixed points of the DE) is that a transcritical or subcritical bifurcation of the types shown in Fig. 3.19 exists at the bifurcation point or near a bifurcation limit point. It is emphasized here that the existence of spurious fixed points of higher period can be independent of the existence of spurious steady states (fixed points of period 1) attached to (or bifurcated from) the main branch $1_{E}$.

A detailed analytical study on the existence of transcritical, subcritical, and supercritical bifurcations for the class of Runge-Kutta methods can be found in our companion paper [13]. Figures 3.13-3.15 illustrate the onset of different types of spurious steady-states, by showing the stable and unstable fixed points of periods 1 and 2, and the types of bifurcation phenomena for the modified Euler, improved Euler, and R-K 4, and the predictor-corrector schemes of orders 2 and 3 for $S(u)=$ $u(1-u)$ and $S(u)=u(1-u)(b-u), 0<b \leqslant 0.5$. In order to illustrate the different behavior in an uncluttered fashion, not all of the periods 1 and 2 and branching points are labelled. It is interesting to see the manner in which the onset of the different types of bifurcations occur, in particular, the birth of the different types of bifurcations away from the $1_{E}$ branches. With the aid of the stable and unstable fixed point diagrams, one can identify the type of bifurcation phenomena easily.

\subsection{Basins of Attraction}

Due to the different dependence on and sensitivity to initial data of the individual DEs and the discretized counterparts, in conjunction with the existence of spurious steady states and asymptotes of higher periods, even associated with the same (common) steady-state solution, the basin of attraction of the continuum might be vastly different from that of the discretized counterparts.

Take for example, $S(u)=u(1-u)$. The only stable fixed point of the logistic ODE is $u=1$ for any positive $\alpha$. The entire domain of the real $u^{n}$-axis is divided into two basins of attraction for the ODE independent of any real $\alpha$. Now if one numerically integrates the ODE by the modified Euler method, extra stable and unstable fixed points can be introduced by the scheme depending on the value of $r$. That is 
for certain ranges of the $r$ values, the $u^{n}$-axis is divided into four basins of attraction. But, of course, for other ranges of $r$, higher period spurious numerical solutions exist, more basins of attraction are created within the same $u^{n}$-axis range, etc. Stable and unstable fixed point diagrams such as Figs. 3.13-3.15 are usually very useful in the division of the $u^{n}$-axis into different basins of lower periods. The unstable fixed points "lines" (period 1 or higher periods) usually form the boundaries of the basins. The basins of attraction for the various methods were computed and colored according to the lower order period. They are not shown here due to the lack of color printing.

\subsection{Systems of $O D E S$}

As can be seen from the previous sections, the rich and complicated dynamical behavior of discrete maps resulting from finite discretization of simple nonlinear scalar autonomous ODEs is enlightening, educational, and useful in giving some indications of the strange behavior encountered in practice. One would naturally ask how highly coupled nonlinear first-order autonomous systems complicate the issue. After all, these types of systems occur naturally in physical science and engineering fields. Examples are

(1) nonlinear scalar first or $m$ th-order nonautonomous ODEs arising from physical, biological, and engineering sciences,

(2) second or higher order nonlinear scalar autonomous ODEs arising from physical, biological, and engineering sciences,

(3) turbulence and turbulence modeling in fluid dynamics [87-89],

(4) meteorology,

(5) chemical reaction equations arising from chemistry,

(6) system of ODEs arising from the method of lines approach in PDEs such as the reaction-diffusion, reaction-convection, and reaction-convection-diffusion equations.

Future work will be directed towards investigation into the nonlinear dynamical effect of using ODE solvers for nonlinear system of ODEs. Here, we do not attempt to give a detailed discussion on this subject, but rather indicate some of the implications from our experience as well as from what is availiable in the literature.

First, the coupling of first-order nonlinear systems arising from a higher-order scalar nonlinear ODE is very different from the truly nonlinear coupling on systems of first-order ODEs. This difference carries over to their discretized counterparts. Second, due to the nonlinear coupling effect, most of the phenomena that are observed in the nonlinear scalar case will most likely carry over to the coupled system case in a more complex manner. Even with help of the center manifold theorem [3-6], nonlinear systems of higher than three first-order ODEs are still 
extremely difficult to analyze. One major factor in analyzing the associated discrete maps is that when three or more time levels of ODE solvers are used, even though the continuum is a first-order scalar autonomous ODE, the resulting discrete maps are $(p-1)$ th-order, where $p$ is the time level. One can extrapolate the complexity involved if nonlinear coupled systems of higher-order ODEs were discretized by $p$-time levels of ODE solvers. Some aspects and implications of numerical integration of second- and third-order ODEs are discussed in Refs. [51, 52, 90]. Some of our numerical experiments agree with the above general conclusion.

In our companion paper [14], the dynamical behavior of the previously studied ODE solvers on $2 \times 2$ systems of autonomous ODEs is investigated. In depth global analysis similar to the level of the scalar case is very involved and is extremely difficult to analyze. The intent of our companion paper is to gain a first-hand understanding of the subject. The most interesting result is that due to higher dimensional nonlinear coupling effects, stable spurious steady states occurring below the linearized stability limit were not observed on the five different coupled $2 \times 2$ nonlinear ODE systems. However, more complex phenomena such as stable spurious limit cycles and stable spurious higher dimensional tori were observed. The study in Ref. [14] indicates that all of the studied Runge-Kutta methods exhibit spurious limit cycles. We hypothesize that the existence of spurious limit cycles and higher dimensional tori might be one of the major contributing factors in slow convergence or nonconvergence in the use of the time-dependent approach to the steady states in practical computations.

\section{LEVEL OF COMPLEXITY FOR PDES}

At present few general results are known on the dynamics of numerics for nonlinear nonhomogeneous hyperbolic and parabolic PDEs. In order to gain some insight into this area, we shall be content with pursuing the subject in three stages. First, we will attempt to obtain a better understanding of the subject of time discretization of ODEs. The investigation can give insight into numerical methods employing the Strang type of operator splittings [91] or methods of lines [92] approach for nonhomogeneous hyperbolic and parabolic PDEs. The second stage will involve the study of the discrete traveling wave solutions of the reactionconvection and reaction-convection-diffusion equations. The third stage will involve the study of the complete temporal-spatial discretizations of the reaction-convection and reaction-convection-diffusion equations. The last stage of the proposed plan is extremely difficult to analyze. Some aspects of full discretizations, traveling wave solutions, and discrete traveling wave solutions were investigated in $[10,58-66$, 68-71, 93-96]. More recent results are reported in our companion papers [15-18].

The following is an attempt to give a flavor of the subject and at the same time provide a justification for the importance of this subject area in CFD algorithm development for our next generation aerodynamics needs. 


\subsection{Model Equations}

One of the recent areas of emphasis in CFD has been the development of appropriate finite-difference methods for combustion and nonequilibrium gas dynamics in the hypersonic range [2, 21, 97-99]. A nonlinear scalar reactiondiffusion model equation would be of the form

$$
\frac{\partial u}{\partial t}=\varepsilon \frac{\partial^{2} u}{\partial x^{2}}+\alpha S(u), \quad \varepsilon, \alpha \text { system parameters }
$$

a nonlinear scalar reaction-convection model equation would be of the form

$$
\frac{\partial u}{\partial t}+\frac{\partial f(u)}{\partial x}=\alpha S(u)
$$

and a nonlinear scalar reaction-convection-diffusion model equation would be of the form

$$
\frac{\partial u}{\partial t}+\frac{\partial f(u)}{\partial x}=\varepsilon \frac{\partial^{2} u}{\partial x^{2}}+\alpha S(u)
$$

Here $f(u)$ is a linear or nonlinear function of $u$. The nonlinear source term (or the reaction term) $S(u)$ can be very stiff. Note that phenomena such as chaos, bifurcations and limit cycles most often relate to source terms $S(u)$ which are nonlinear in $u$. Equation (4.3) can be viewed as a model equation in combustion or as one of the species continuity equations in nonequilibrium flows (except in this case, the source term is coupled with other species mass fractions).

\subsection{Level of Complexity}

As in the ODE case, the goal is to investigate what types of new phenomena arise from the numerical methods that are not present in the original nonlinear PDE, as a function of the stiff coefficient $\alpha$, the diffusion coefficient $\varepsilon$, and the time step $\Delta t$ with a fixed (or variable) grid spacing $\Delta x$. The time step can vary greatly depending on whether the time discretization is explicit or implicit. The study can be divided into steady and unsteady behavior with or without shock waves.

In addition to the fact that spurious equilibrium states can be introduced by the time differencing and/or the spatial differencing, combustion-related and high speed hypersonic flow problems usually contain multiple equilibrium states and shock waves that are inherent in the governing equations. In many instances the stable and unstable equilibrium states, whether due to the physics or natural spuriousness, are interwoven over the domain of interest and are usually highly dependent on the initial conditions and the time steps (even when the chosen time step is within the linearized stability limit as indicated in our study), as well as variation of parameters such as angle of attack, Reynolds number, and coefficients of physical 
and numerical dissipations and physical and numerical boundary conditions. One can extrapolate the complexity involved when the influence of the various temporal, as well as spatial, discretizations are sought on the basins of attractivity.

The sensitivity of numerical solutions to coefficients of physical and numerical dissipation is evident from the study of Mitchell and Bruch [59] on the reactiondiffusion equation. Their main result is that diffusion, which is usually perceived as having a stabilizing effect, is able to produce chaotic as well as divergent numerical solutions. Another interesting result due to Mitchell and Bruch was the production of chaos by decreasing the space increment or increasing the time increment. They showed that the addition of diffusion poses severe problems unless waves of constant speed $c$ are assumed, in which case it reverts to an ODE with $x+c t$ as the independent variable. The sensitivity of numerical solutions to numerical boundary condition procedures was discussed in $[100,101]$.

In order to assess the possible errors, slow convergence and nonconvergence of steady-state numerical solutions when using the time-dependent approach for nonlinear reaction-convection BVPs, four different numerical aspects are addressed in our companion papers $[17,18]$. First, they show that stable and unstable spurious steady-state numerical solutions (numerically irrelevant solutions) can be independently introduced by spatial and temporal discretizations satisfying the same boundary condition and initial data. Second, they investigate how the various ways of discretizing the reaction term can drastically affect the stability of the spurious as well as the exact steady-state solutions. Third, they illustrate how the time discretization can destablize the stable spurious steady-state numerical solutions that are introduced by the spatial discretizations or vice versa. Finally, they show how the numerical phenomenon of incorrect propagation speeds of discontinuities may be linked to the existence of some stable spurious steady-state numerical solutions.

The results in Refs. $[17,18]$ are concerned with separable temporal and spatial finite difference approximations (as those arising from the method of lines resulting in semi-discrete approximations of the PDE). Their illustrations of the existence of spurious steady states for the semi-discrete approximations serve a dual purpose. That is, their study also indicates the existence of spurious steady-state numerical solutions when the steady-state PDE, rather than the time-dependent approach, is used to solve for the steady-state numerical solution. A major difference in the dynamical behavior between the two approaches to obtaining steady-state numerical solutions is that for the time-dependent approach, the time discretization can independently contribute spurious steady states, but at the same time it can destablized the spurious steady states that are introduced by the spatial discretization or vice versa. See [16-18] for a discussion. The interplay between the spatial and time discretizations on the stability of spurious solutions (by both time and spatial discretizations) is very complex. At this time few general results are known. On the other hand, by solving the steady-state PDE, all spurious steady states due to the spatial discretizations are present. There is no concept of stability since there is no time involved. The difficulty lies not only in the method of identifying the 
TABLE II

Systematic Approach-Level of Complexity

I. ODE Connection: Gain insight into time discretization of PDEs

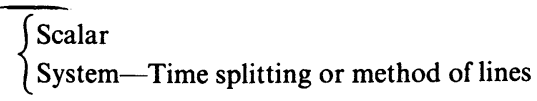

II. Discrete travelling wave:

$$
\begin{aligned}
& \frac{\partial u}{\partial t}+C \frac{\partial u}{\partial x}=\varepsilon \frac{\partial^{2} u}{\partial x^{2}}+\alpha S(u) \\
& \text { Scalar }\left\{\begin{array}{l}
\text { Reaction-diffusion } \sqrt{ } \\
\text { Reaction-convection } \sqrt{ } \\
\text { reaction-convection-diffusion }
\end{array}\right.
\end{aligned}
$$

III. Full Discretization (temporal and spatial):

$$
\begin{aligned}
& \text { Scalar }(S \neq 0)\left\{\begin{array}{l}
-\sqrt{ } / \\
-\sqrt{ }
\end{array}\right\} \text { linear scheme for spatial discretization } \\
& \text { Scalar }(S=0)\left\{\begin{array}{l}
- \\
-
\end{array}\right\} \text { nonlinear scheme for spatial discretization } \\
& \text { Scalar }(S \neq 0)\left\{-\frac{}{\square}\right\} \text { nonlinear scheme for spatial discretization }
\end{aligned}
$$

spurious steady states from the exact ones, but also in the major shortcoming that quite often one has to deal with PDEs of the mixed type.

Table II summarizes the level of complexity for a systematic approach to these types of PDE. The check mark on each type of PDE and approach indicates the ones where some work has been done on this subject.

\subsection{Involvement in the Study of Full Discretization of PDE}

Consider a three-level explicit time differencing and a three-point spatial differencing of the reaction-convection-diffusion equation (4.3) of the form

$$
u_{j}^{n+1}=u_{j}^{n}+H\left(u_{j-1}^{n}, u_{j}^{n}, u_{j+1}^{n}, u_{j-1}^{n-1}, u_{j}^{n-1}, u_{j+1}^{n-1}, \alpha, \varepsilon, \Delta t, \Delta x\right)
$$

where $u_{j}^{n}$ is the numerical solution at $t=n \Delta t$ and $x=j \Delta x$. Then the study of the asymptotes of (4.4) amounts to the study of fixed point behavior of period $p$ in time and period $q$ in space, denoted by $(p, q)$, where $p$ and $q$ are integers. Here the fixed point of the partial-difference equation (4.4) is defined in a slightly more complicated way than for the ODE. 
For example, a fixed point of period $(1,1)$ is defined as $u_{j+1}^{n+1}=u_{j}^{n}$ and a fixed point of period $(2,1)$ is defined as $u_{j+1}^{n+2}=u_{j}^{n}$. However, a fixed point of period $(1,2)$ is defined as $u_{j+2}^{n+1}=u_{j}^{n}$. Thus, in general, a fixed point of period $(p, q)$ is defined as $u_{j+q}^{n+p}=u_{j}^{n}$. One can see that for $p, q>3$, solving the resulting nonlinear algebraic equation is very involved, especially when physical boundary conditions and physical dissipation terms as well as numerical boundary conditions $[100,101,22,17$, 18] and numerical dissipation [59] are additional dimensions of consideration. Current available work involving the studies beyond the linearized stability limit of the schemes, and assuming the nonexistence of spurious fixed points of period $(1,1)$ for reaction diffusion equations and reaction convection equations are reported in Refs. [20, 38, 58-66, 68-71]. Some issues on spurious steady-state numerical solutions are discussed in [102] and our companion papers $[17,18]$.

\subsection{Influence on Dynamical Behavior by Property of the PDEs and Schemes, and Treatment of the Source Terms}

Although the general study of the dynamical behavior of partial-difference equation for (4.3) is an enormous task, if we can isolate certain restricted subsets of the PDEs and schemes in hand which are immune to the type of phenomena discussed in Section III for time discretization as well as spatial discretization, then we can concentrate on the rest of the unknowns. For a comprehensive introduction to conservation laws, see Refs. [103, 104].

As can be seen in Section III, the nature of the dynamical behavior of the discretized counterparts is strongly influenced by properties of the numerical method and the types and form of nonlinear DEs. Here we want to study the influence on the dynamical behavior of elements such as conservation and nonlinearity of the schemes and treatment of the source terms [2, 35-37, 21, 97-99] when nonlinear conservation laws are sought.

First, take the scalar convection equation (4.2) with $S(u)=0$ and consider a conservative explicit scheme $[105,106,2]$ whcih is consistent with the conservation law of the form

$$
u_{j}^{n+1}=u_{j}^{n}-\lambda\left[h_{j+1 / 2}^{n}-h_{j-1 / 2}^{n}\right],
$$

where $\lambda=\Delta t / \Delta x$ and $h_{j \pm 1 / 2}^{n}$ are the numerical flux functions. For a two-time level and five-point spatial scheme, $h_{j \pm 1 / 2}^{n}=h\left(u_{j}^{n}, u_{j \pm 1}^{n}, u_{j \pm 2}^{n}\right)$.

We also can consider a two-parameter family of schemes

$$
\begin{aligned}
u_{j}^{n+1}+\frac{\lambda \theta}{1+\omega}\left[h_{j+1 / 2}^{n+1}-h_{j-1 / 2}^{n+1}\right]= & u_{j}^{n}-\frac{\lambda(1-\theta)}{1+\omega}\left[h_{j+1 / 2}^{n}-h_{j-1 / 2}^{n}\right], \\
& +\frac{\omega}{1+\omega}\left(u_{j}^{n}-u_{j}^{n-1}\right),
\end{aligned}
$$

where $0 \leqslant \theta \leqslant 1$. When $\theta=0$, the scheme is explicit and when $\theta=\omega+\frac{1}{2}$, the scheme 
is temporally second-order accurate. One can obtain (4.5) from (4.6) by setting $\theta=0$ and $\omega=0$. The time differencing belongs to the class of LMM. Under the assumption that this scheme is conservative and consistent with the 1D conservation law, discrete map (4.6) will have no spurious steady-state numerical solution, since consistency means

$$
h\left(u^{*}, u^{*}, u^{*}\right)=f\left(u^{*}\right) .
$$

Thus any steady-state solutions of (4.6) are steady-state solutions of the original PDE. The above result assumes no nontrivial exact steady state of the conservation law exists. However, this does not preclude (4.7) from the possibility of exhibiting spurious limit cycles that can interfere with stability, convergence, and basins of attraction of the true physics of the continuum. See our companion paper [14] or Section 4.6 for a short discussion. We remark that the situation is more complicated if higher than 1D conservation laws $(S=0)$ are considered since nontrivial exact steady states exist.

Now the situation is different when $S(u) \neq 0$. Under this solution, even if the same time and spatial discretization are employed, one still has to evaluate $\bar{S}$ properly. Here $\bar{S}$ is the function $S$ evaluated at some proper average state $\bar{u}$ $[2,35-37]$ for the full discretization that is consistent with the scheme and achieves conservation at jumps. For a discussion on this subject, see Refs. [21, 2, 35-37] for details. The other crucial aspect is that when $S(u) \neq 0$, a full investigation into the dynamical behavior of the temporal and spatial discretization is necessary. The knowledge gained from the finite-difference methods analysis for $S(u)=0$ does not carry over to the $S(u) \neq 0$ case $[14,17,18]$.

\subsection{Discrete Traveling Waves}

Analysis of the dynamical behavior of the full discretization of nonlinear nonhomogeneous PDEs of the hyperbolic and parabolic types is very involved. In this section, we look at a more restricted class of solutions-the discrete traveling wave solutions.

Consider a reaction-diffusion equation

$$
\frac{\partial u}{\partial t}=\frac{\partial^{2} u}{\partial x^{2}}+S(u)
$$

Solution $u(x, t)$ depends on the space variable $x$ and on the time $t$. Every zero of $S(u)$ constitutes an equilibrium of the PDE. Then a traveling wave solution is a profile $U(x)$ that travels along the $x$-axis with propagation speed $\bar{\lambda}$. Neither the shape of the wave nor the speed of propagation changes. To find traveling waves, we seek solutions

resulting in an ODE

$$
u(x, t)=U(x-\bar{\lambda} t)
$$

$$
U^{\prime \prime}+\bar{\lambda} U^{\prime}+S(U)=0
$$


By solving this ODE, one can calculate asymptotic states for the PDE. Let $u_{1}$ and $u_{2}$ be roots of $S(u)$ and hence equilibrium solutions for both the PDE and the ODE. The asymptotic behavior of solution $U$ for $x \rightarrow \pm \infty$ determines the type of traveling wave. Every solution with

$$
\begin{aligned}
U(\infty) & =u_{1} \\
U(-\infty) & =u_{2},
\end{aligned}
$$

with $u_{1} \neq u_{2}$, is a front wave of the ODE. This corresponds to a heteroclinic orbit [4] of the ODE, connecting the two stationary points $u_{1}$ and $u_{2}$. Here for a secondorder autonomous ODE (4.10), when distinct saddles are connected, one encounters a heteroclinic orbit; also a heteroclinic orbit may join a saddle to a node or vice versa. Another type of special orbit is a homoclinic orbit. A homoclinic orbit connects a saddle point to itself and such orbits have an infinite period. Several heteroclinic orbits may form a closed path called a homoclinic cycle. Both the heteroclinic and homoclinic orbits are of great interest in applications because they form the profiles of traveling wave solutions of many reaction-diffusion problems. See Refs. $[4,11,93,94]$ for a discussion.

Similarly, one can study discrete traveling wave solutions for the finite discretization of (4.8). See Refs. [93-96] for a discussion. Before a short description of the subject, it is important to point out that understanding of the discrete traveling wave solutions of the corresponding PDEs only gives insight into a very small subset of the dynamics of the PDEs. In most cases, it provides no information at all for the fully discretized equation. Although there is a considerable body of knowledge concerning traveling wave solutions of (4.8), little work has been done for the corresponding discrete case. A recent study by Keener [95] concerns the semi-discrete equations

$$
\frac{d u_{j}}{d t}=\frac{\left[u_{j-1}-2 u_{j}+u_{j+1}\right]}{(\Delta x)^{2}}+S\left(u_{j}\right) .
$$

In the case that $S(u)$ is $N$-shaped

$$
\begin{array}{rll}
S(0)=S(b)=S(1)=0, & S^{\prime}(0)<0, & S^{\prime}(1)<0 \\
S(u)<0 \quad \text { for } \quad 0<u<b, & S(u)>0 \quad \text { for } \quad b<u<1 .
\end{array}
$$

A detailed analysis of the corresponding continuous problem is given by Fife and McLeod [96] who proved, among other things, that (4.8) has a unique traveling wave of the form (4.9), (4.11) with $u_{1}=1$ and $u_{2}=0$. A simple example of an $N$-shaped source term is given by

$$
S(u)=u(u-b)(1-u) \quad \text { with } \quad 0<b<\frac{1}{2} .
$$

For the system (4.12), Keener [95] shows that 
1. for $\Delta x$ sufficiently large there is an infinite number of stable nonuniform standing wave solutions of (4.12) and these effectively act to block the propagation of traveling waves,

2. for $\Delta x$ sufficiently small the system exhibits propagation (the technique used is that of super- and sub-solutions but, unfortunately, does not reveal the existence of shape invariant solutions of the form $u_{j}(t)=u(j \Delta x-\gamma t)$ with propagation speed $\gamma$ ), and

3. monotone shape invariant traveling waves, if they exist, are stable.

In the case of the cubic source term it is shown that propagation is blocked if $b \Delta x>2$, whereas propagation is assured for Heaviside initial data if

$$
(\Delta x)^{2}<\frac{25}{2 b^{2}-b+1-2(1+b) \sqrt{b^{2}-3 b+1}},
$$

provided $b^{2}-3 b+1>0$, or $0<b<0.382$. Numerical experiments suggest that propagation first occurs at a value of $\Delta x$ between the given bounds.

The simplest fully discrete approximation of $(4.8)$ is given by

$$
u_{j}^{n+1}=u_{j}^{n}+\bar{r}\left[u_{j-1}^{n}-2 u_{j}^{n}+u_{j+1}^{n}\right]+\Delta t S\left(u_{j}^{n}\right),
$$

where $\bar{r}=\Delta t / \Delta x^{2}$. The behaviour of this scheme for $S(u)=\alpha u(1-u)(\alpha>0)$ in the neighbourhood of its fixed points $u^{*}=0,1$ has been studied extensively (see $[59,65,68])$. It has been shown that solutions of $(4.16)$ are stable in the neighborhood of $u^{*}=1$ (the stable fixed point of $(4.8)$ ) provided that

$$
0<\alpha \Delta t<2(1-2 \bar{r})
$$

which defines the linearized stability limit of the method. When the rightmost inequality is violated, the constant solution $u_{j}^{n}=1$ bifurcates and the system admits solutions of the form $u_{j}^{n}=$ const $+\varepsilon(-1)^{n-j}$ for some amplitude $\varepsilon$. This type of solution represents a wave of wavelength $2 \Delta x$ that travels a distance $\Delta x$ during each time step.

The study of shape invariant traveling wave solutions of (4.16) has to be restricted to situations where the speed of propagation is a rational multiple, $q / p$ say, of the mesh ratio $\Delta x / \Delta t$. Uniform waves of this type correspond to the period $(p, q)$ solutions discussed in [65] where it was deduced that, for each value of $\Delta t$ where the logistic equation (3.12) has a stable solution of period $p,(4.16)$ admits a stable solution of period $(p, 1)$ for $\bar{r}$ sufficiently small.

To illustrate some of the aspects involved, we consider shape invariant waves that travel one grid point per time step. That is, they are more general forms of period $(1,1)$ solutions and are characterized by $u_{j}^{n}=v_{n-j}$ for all $n$ and $j$. Substituting this into (4.16) leads to the ordinary difference equation

$$
v_{j-1}=\left[(1-2 \bar{r}) v_{j}+\bar{r} v_{j+1}+\Delta t v_{j}\left(1-v_{j}\right)\right] /(1-\bar{r}), \quad j=\cdots, 1,0,-1,-2, \cdots,
$$


which has been written as a backward recursion so that the fixed point $u^{*}=1$ is stable (an attractor) provided that (4.17) holds. Thus, if a sequence $v_{j}$ is constructed satisfying (4.18) and this is used as initial data for (4.12) $\left(u_{j}^{0}=v_{j}\right)$, then the resulting solutions of (4.12) will be traveling waves with the required property. Numerical evidence suggests that these are stable, subject to (4.17), but there is, to our knowledge, no theoretical analysis available. It should be noted that (4.18) is a consistent linear multistep method for the differential equation

$$
\frac{d v}{d t}=-\alpha v(1-v)
$$

solved backwards in time.

When the right inequality in (4.17) is violated, the solutions of (4.18) undergo a period doubling bifurcation so that $v_{j}$ tends to constant $+\varepsilon(-1)^{j}$ as $j$ tends to $-\infty$. As $\Delta t$ increases, say, for fixed $\bar{r}$, further bifurcations occur, including the appearance of strange attractors, until the solution eventually escapes to $\infty$ (blows up). The association with strange attractors can be made more precise by the change of variable

$$
v_{j}=\left[\frac{1}{2}+\frac{1-2 \bar{r}}{2 \Delta t}\right]+\frac{\Delta t}{1-\bar{r}}\left[\frac{1}{4}-\left(\frac{1-2 \bar{r}}{2 \Delta t}\right)^{2}\right] H_{j} .
$$

In this case, (4.18) becomes

$$
H_{j-1}=1-\mu H_{j}^{2}+\beta H_{j+1},
$$

the Henon map [107] written as a backward recursion, where

$$
\mu=\left(\frac{\Delta t}{1-\bar{r}}\right)^{2}\left[\frac{1}{4}-\left(\frac{1-2 \bar{r}}{2 \Delta t}\right)^{2}\right], \quad \beta=\frac{\bar{r}}{1-\bar{r}} .
$$

The most common choice of parameters in the Henon map are $\mu=1.4, \beta=0.3$ which correspond to $\bar{r}=0.321$ and $\Delta t=1.898$.

Similar arguments can be applied to hyperbolic equations with nonlinear source terms, but work in this area is still at a very early stage and the dynamical behavior of the discrete traveling waves is more complicated to analyze. The reader is referred to our companion paper [15] for a discussion.

\subsection{Spurious Limit Cycles vs Slow Convergence or Nonconvergence of Numerical Methods}

For the purpose of illustration, we consider the viscous Burgers' equation with zero source term

$$
\frac{\partial u}{\partial t}+\frac{1}{2} \frac{\partial\left(u^{2}\right)}{\partial x}=\varepsilon \frac{\partial^{2} u}{\partial x^{2}}, \quad \varepsilon>0
$$


Let $j=1, \ldots, J$. We consider a three-point central difference discretization in space with periodic condition $u_{j}=u_{J+j}$, which implies that $\sum_{j=1}^{J}\left(d u_{j} / d t\right)=0$ or $\sum_{j=1}^{J} u_{j}=$ const. This system is sometimes referred to in the literature as a perturbed Hamiltonian. For simplicity, take $J=3$ and $\Delta x=\frac{1}{3}$. Then

$$
\begin{aligned}
\frac{d u_{1}}{d t}+\frac{3}{4}\left(u_{2}^{2}-u_{3}^{2}\right) & =\varepsilon\left(u_{2}-2 u_{1}+u_{3}\right) \\
\frac{d u_{2}}{d t}+\frac{3}{4}\left(u_{3}^{2}-u_{1}^{2}\right) & =\varepsilon\left(u_{3}-2 u_{2}+u_{1}\right) \\
\frac{d u_{3}}{d t}+\frac{3}{4}\left(u_{1}^{2}-u_{2}^{2}\right) & =\varepsilon\left(u_{1}-2 u_{3}+u_{2}\right) \\
\sum_{j=1}^{3} \frac{d u_{j}}{d t} & =0 .
\end{aligned}
$$

This system can be reduced to a $2 \times 2$ system of first-order nonlinear autonomous ODEs. In this case, the nonlinear convection term is contributing to the nonlinearity of the ODE system (4.24). Equation (4.24) has four steady-state solutions (fixed points) of which three are saddles and one is a stable spiral at $\left(\frac{1}{3}, \frac{1}{3}\right)$ for $\varepsilon \neq 0$. For $\varepsilon=0$ the stable spiral becomes a center.

To study the bifurcation phenomena of the corresponding steady-state numerical solutions of time discretizations of the semi-discrete system (4.24) as the time step is varied, two existing interactive computer programs were modified and adopted for our current computations. One of the interactive computer programs, written by Creon Levit of NASA Ames, was originally designed to aid the study of flow visualization in CFD. The other computer program "AUTO" [108], written by Eusebius Doedel, is a software package for continuation and bifurcation problems in ODEs.

The time discretizations that we are considering are similar to our scalar study. These schemes include the explicit Euler, modified Euler, improved Euler, Heun (a third-order Runge-Kutta method), and a fourth-order Runge-Kutta method. Our studies indicate that all of the studied Runge-Kutta methods exhibit spurious limit cycles. See our companion paper [14] for more details.

Note that spurious steady states are not a problem for this model PDE (4.23), since $S=0$ and LMM time discretizations and conservative schemes are used. However, the existence of stable and unstable spurious limit cycles makes the basins of attraction of the discretized counterparts very different from the continuum and thus can have severe effects on the speed of convergence or possible nonconvergence of numerical solution from a given set of initial data even though the data may be physically relevant. 


\section{IMPLICATIONS AND RECOMMENDATIONS}

Due to the complexity of the large increase in system dimension and the involvement of multiple floating parameters for finite difference methods in PDEs, we are not certain that a similar systematic general result can be arrived at for more complex nonlinear systems. The main indication at this point is from our time discretization study and some early stage development for the model reaction-diffusion and reaction-convection PDEs.

\subsection{Results Drawn from the ODE Connection Study and from Our Companion Papers}

Our study illustrates a few fundamental aspects in explaining what happens when linear stability breaks down for truly nonlinear problems, i.e., for equations that display genuinely nonlinear types of behavior. The important points are as follows:

(1) For certain time discretizations, spurious steady-state solutions may occur below the linearized stability limit of the scheme.

(2) The result of operating with a time step beyond the linearized stability limit is not always a divergent solution; spurious steady-state solutions can occur.

(3) Associated with the same (common) steady-state solution the basin of attraction of the DEs might be vastly different from that of the discretized counterparts. This is mainly due to the dependence on and sensitivity to initial conditions and boundary conditions for the individual systems. In the absence of the influence of the initial and boundary conditions, the difference in the basins of attraction between the continuum and its discretized counterparts occurs even when an implicit unconditionally stable LMM type of method is used unless the resulting nonlinear algebraic equations are solved exactly.

(4) Nonunique steady-state solutions can be introduced by the spatial discretization even though the original PDEs might possess only a unique steady-state solution and a LMM type of time discretization is used so that no spurious steady state can be introduced by time discretizations $[17,18]$. The tie between temporal and spatial dynamical behavior is more severe when one is dealing with the nonseparable temporal and spatial finite-difference discretization such as the Lax-Wendroff type, where the time and spatial difference cannot be separated from each other [16]. The situation would be more complicated if the governing nonlinear PDE possessed more than one steady-state solution as well as the spurious ones that are purely due to the numerical method.

(5) Spurious limit cycles can be generated by finite discretizations of nonlinear PDEs containing zero source terms. The existence of stable spurious limit cycles might be one of the contributing factors in nonconvergence of the timedependent approach to the steady state. 
(6) There is a misconception that computational instability or inaccuracy can be cured simply by making $\Delta t$ smaller. Other elements such as (1)-(5) above, as well as the variation of the grid spacings, numerical dissipation terms, and system parameters other than the time steps can interfere with the dynamical behavior. See, for example, Fig. 3.6 for the case of the logistic ODE and the modified Euler method. Figure 3.6 shows that if the initial data is not inside the basins of attraction for the $1_{E}$ branch of the solution, no matter how one reduces the time step, the numerical solution will not converge to the exact steady state.

(7) When linearized stability limits are used as a guide for a time step constraint for highly coupled nonlinear system problems, this time step might exceed the actual linearized stability limit of the coupled equations. Therefore all of the situations in (1)-(6) can occur. In particular, when one tries to stretch the maximum limit of the linearized allowable time step for highly coupled systems, most likely all of the different types of spurious branches of supercritical, subcritical, and trancritical bifurcations can be achieved in practice, depending on the initial conditions. Consequently, the occurrence of spurious steady-state solutions beyond the linearized stability limit is not just secondary, but might be as important as the occurrence of spurious steady states below the linearized stability limit. This is compounded in practical situations where the exact linearized stability is not usually computed, but rather a frozen coefficient procedure at each time step with a fixed grid spacing used to estimate the stability limit of the algorithm. Therefore, in practical computations, erroneous numerical can easily be achieved unknowingly.

(8) The occurrence of spurious asymptotes is independent of whether the DE possesses a unique steady state or has additional periodic solutions and/or exhibits chaotic phenomena. The form of the nonlinear DEs and the type of numerical schemes are the determining factors.

(9) It is not just the occurrence of stable spurious numerical solutions that causes difficulty. Indeed such cases may be easier to detect. These spurious features of the discretizations often occur but can be unstable; i.e., they do not appear as an actual (spurious) solution because one usually cannot obtain an unstable asymptotic solution by mere time integration. However, far from being benign, they can have severe detrimental effects on the basins of attraction of the true solution for the particular method, hence causing slow convergence or possibly even nonconvergence from a given set of initial data even though the data might be physically relevant.

\subsection{Recommendations}

Although more theoretical development and better guidelines are needed to aid the construction of appropriate algorithms for PDEs containing nonlinear source terms and although the understanding of the topic is still at an early stage, nevertheless, we believe nonlinear dynamics play a vital role in this research area. 
In spite of the limited knowledge in hand, we believe it is of importance to know the nonlinear dynamical behavior of the various schemes before their actual use for practical applications. Otherwise, it might be very difficult to assess the accuracy (spurious or otherwise) of the solution when the numerical method is the sole source of the understanding of the physical solutions. When in doubt, it is always safer to use schemes that do not produce spurious steady-state solutions for the nonlinear scalar case. Some examples of methods of this type in time discretization can be listed:

(1) LMM [27] ODE solvers such as the explicit, implicit Euler, three-point backward differentiation, etc. can be used.

(2) One can use the "regular" Runge-Kutta methods [28, 29].

(3) Solving the nonlinear algebraic systems arising from implicit LMM exactly would avoid spurious steady-state numerical solutions. Otherwise, the type of iteration method used in solving nonlinear algebraic systems can degrade the basin of attractivity of implicit LMM [27, 28].

The insight gained from time discretization will only give an indication in separable schemes or method of lines approaches. Also, the commonly used residual test [109-111] in the time-dependent approach to the steady state might be misleading. This is the direct consequence of what was indicated in Section 5.1. The procedure of using the inverse problem of nonlinear dynamics to analyze time series data from a finite difference method computer code in an attempt to learn about the true behavior of the solution of the continuum governing PDEs without knowing by other means the exact solution behavior of the PDEs other than the numerical solutions can yield misleading results. These will be discussed in the next two sections.

\subsection{Residual Test}

Consider a quasilinear PDE of the form

$$
\frac{\partial u}{\partial t}=G\left(u, u_{x}, u_{x x}, \alpha, \varepsilon\right),
$$

where $G$ is nonlinear in $u, u_{x}$, and $u_{x x}$ and $\alpha$ and $\varepsilon$ are system parameters. For simplicity, consider a two-time level and a $(p+q)$-point grid stencil of the form

$$
u_{j}^{n+1}=u_{j}^{n}-H\left(u_{j+q}^{n}, \ldots, u_{j}^{n}, \ldots, u_{j-p}^{n}, \alpha, \varepsilon, \Delta t, \Delta x\right)
$$

for the PDE (5.1). Let $U^{*}$, a vector representing $\left(u_{j+q}^{*}, \ldots, u_{j}^{*}, \ldots, u_{j-p}^{*}\right)$, be a steadystate numerical solution of (5.2). It is a common practise in CFD to use a time-dependent approach such as (5.2) to solve the steady-state equation $G\left(u, u_{x}, u_{x x}, \alpha, \varepsilon\right)=0$. The iteration is stopped when the residual $H$ and/or some $L_{2}$ 
norm of the dependent variable $u$ between two successive iterates is less than a pre-selected level.

Aside from the various standard numerical errors such as truncation error, machine round-off error, etc. [112], there is a more fundamental question on the validity of the residual test and/or $L_{2}$ norm test. If the scheme happens to produce spurious steady-state numerical solutions (due to spatial discretizations), these spurious solutions would still satisfy the residual and $L_{2}$ norm tests in a deceptively smooth manner. Moreover, aside from the spurious solutions issue, depending on the combination of time as well as spatial discretizations, it is not easy to check whether $G\left(u^{*}, u_{x}^{*}, u_{x x}^{*}, \alpha, \varepsilon\right) \rightarrow 0$ even though $H\left(U^{*}, \alpha, \varepsilon, \Delta t, \Delta x\right) \rightarrow 0$, since spurious steady states can be introduced by spatial discretizations. This is contrary to the ODE case, where if $u^{*}$ is spurious in (1.1) then $S\left(u^{*}\right) \neq 0$. Among other factors, this is one of the contributing factors for the increase in magnitude of difficulty when analyzing the dynamical behavior of numerical methods for hyperbolic and parabolic PDEs. See our companion papers $[17,18]$ for a discussion.

One might argue that one can judge the accuracy of the scheme by comparing the numerical solutions with more than one numerical method, and by doing a sequence of grid refinements and time step reductions. The latter approach, however, might not be feasible at an acceptable cost, and the former might not be foolproof if one does not know the dynamical behavior of the finite difference schemes being used. One important contributing factor when using the Lax-Wendroff types of schemes $[113,114]$ is that these schemes are more accurate and sometimes more stable when operated on or near the linearized stability limit, and thus hinder the time step reduction strategy.

\subsection{The Inverse Problems of Nonlinear Dynamics}

The use of the inverse problem of nonlinear dynamics to analyze the dynamical behavior of time series data arising from experimental or observable data has received much attention in nonlinear physics as well as in many of the engineering disciplines. The approach is very useful for gaining some insights into the nonlinear dynamical behavior in problems where experimental or observable data are the main source of information. Often the associated governing equations (continuum or otherwise) do not exist to start with. There has been an explosion of theory, numerical procedures, and computer software addressing this rapidly growing direction [115-118]. There also has been much recent interest in forecasting algorithms that attempt to analyze a time series by fitting nonlinear models. The attractive feature of this approach is that when used correctly on the appropriate problems one can reduce the complexity of the problem from unmanageable higher dimensions to a very low dimension. It is therefore a natural tendency for practioners in computational sciences to apply this approach to analyze the dynamical behavior of time series data from a finite difference method computer code in an attempt to learn about the true physical behavior of the governing PDEs. This application of time series analysis can be misleading and can lead 
to a wrong conclusion if the practitioner does not know by other means the exact solution behavior of the PDEs other than from the numerical solutions. Examples of the use of this type of approach in CFD computations have been presented in Refs. [119-121]. It can be seen from our study that the conclusions drawn from this type of time series analysis provide very little information, but rather can actually mislead one as to the true physics of the problem.

\section{CONCLUding Remarks}

Spurious stable as well as unstable steady-state numerical solutions, spurious asymptotic numerical solutions of higher period, and even stable chaotic behavior can occur when finite-difference methods are used to solve nonlinear DEs numerically. The occurrence of spurious asymptotes is independent of whether the DE possesses a unique steady state or has additional periodic solutions and/or exhibits chaotic phenomena. The form of the nonlinear DEs and the type of numerical schemes are the determining factors. In addition, the occurrence of spurious steady states is not restricted to the time steps that are beyond the linearized stability limit of the scheme. In many instances, it can occur below the linearized stability limit. Therefore, it is essential for practitioners in computational sciences to be knowledgeable about the dynamical behavior of finite-difference methods for nonlinear scalar DEs before the actual application of these methods to practical computations. It is also important to change the traditional ways of thinking and practices when dealing with genuinely nonlinear problems.

In the past, spurious asymptotes were observed in numerical computations but tended to be ignored because they all were assumed to lie beyond the linearized stability limits of the time step parameter $\Delta t$. As can be seen from our study, bifurcations to and from spurious asymptotic solutions and transitions to computational instability not only are highly scheme dependent and problem dependent, but also initial data and boundary condition dependent, and not limited to time steps that are beyond the linearized stability limit.

The symbiotic relation among all of these various factors makes this topic fascinating and yet extremely complex. The main fundamental conclusion is that, in the absence of truncation and machine round-off errors, there are qualitative features of the nonlinear DE which cannot be adequately represented by the finitedifference methods and vice versa. The major feature is that convergence in practical calculations involves fixed $\Delta t$ as $n \rightarrow \infty$ rather than $\Delta t \rightarrow 0$ as $n \rightarrow \infty$. It should be emphasized that the resulting discrete maps from finite discretizations can exhibit a much richer range of dynamical behavior than their continuum counterparts. A typical feature is the existence of spurious numerical asymptotes that can interfere with stability, accuracy, and basins of attraction of the true physics of the continuum. 


\section{ACKNOWLEDGMENTS}

The authors thank J. Yorke and C. Grebogi for their valuable discussions during the course of this research. Special thanks to H. Glaz, P. Gnoffo, M. Hafez, M. Tobak, M. Vinokur, and S. Zalesak for their critical review of the manuscript.

\section{REFERENCES}

1. NASA Computational Fluid Dynamics Conference, NASA Conference Publication 10038, Vols. 1 and 2, March 7-9, 1989.

2. H. C. YeE, NASA TM-101088, Feb. 1989 (unpublished).

3. R. L. DevanY, An Introduction to Chaotic Dynamical Systems (Addison-Wesley, New York, 1987).

4. R. SeYdel, From Equilibrium to Chaos (Elsevier, New York, 1988).

5. J. GuCKenheimer AND P. Holmes, Nonlinear Oscillations, Dynamical Systems, and Bifurcations of Vector Fields (Springer-Verlag, New York, 1983).

6. J. M. T. Thompson and H. B. Stewart, Nonlinear Dynamics and Chaos (Wiley, New York, 1986).

7. C. S. Hsu, Cell-to-Cell Mapping (Springer-Verlag, New York, 1987).

8. M. KubiceK AND M. MAReK, Computational Methods in Bifurcation Theory and Dissipative Structures (Springer-Verlag, New York, 1983).

9. T. S. Parker AND L. O. ChUA, Practical Numerical Algorithms for Chaotic Systems (SpringerVerlag, New York, 1989).

10. E. A. JACKson, Perspectives of Nonlinear Dynamics (Cambridge Univ. Press, Cambridge, UK, 1989).

11. E. Beltrami, Mathematics for Dynamic Modeling (Academic Press, Orlando, FL, 1987).

12. R. H. Abraham and C. D. Shaw, Dynamics-The Geometry of Behavior, Parts I-IV (Aerial Press, Santa Cruz, CA, 1983-1988).

13. P. K. Sweby, H. C. Yee, And D. F. Griffiths, Numerical Analysis Report 3/90, March 1990, University of Reading; NASA TM-102819, April 1990 (unpublished).

14. H. C. Yee AND P. K. Sweby, "Dynamical Approach Study of Spurious Steady-State Numerical Solutions of Nonlinear Differential Equations. Part II. System of $2 \times 2$ ODEs," NASA technical memorandum, 1991 (unpublished).

15. D. F. Griffiths, A. M. Stuart, and H. C. Yee, "Numerical Wave Propagation in Hyperbolic Problems with Nonlinear Source Terms," in preparation.

16. D. F. Griffiths, A. M. Stuart, P. K. Sweby, ANd H. C. YeE, "Stability of Spurious Steady-State Solutions of Runge-Kutta and Related Methods for PDEs," University of Bath internal report, March 1991 (unpublished).

17. A. LAFon AND H. C. YeE, "Dynamical Approach Study of Spurious Steady-State Numerical Solutions of Nonlinear Differential Equations. Part III. The Effects of Boundary Conditions and Nonlinear Source Terms in Reaction-Convection Equations," NASA technical memorandum, in preparation.

18. A. Lafon and H. C. Yee, "Dynamical Approach Study of Spurious Steady-State Numerical Solutions of Nonlinear Differential Equations. Part IV. Stability vs Methods of Discretizing Nonlinear Source Terms," NASA TM-103877, July 1991; submitted for publication.

19. A. C. Newell, SIAM J. Appl. Math. 33, 133 (1977).

20. A. StUART, SIAM Rev. 31, 191 (1989).

21. R. J. LeVeque ANd H. C. Yee, NASA-TM-100075, March 1988; J. Comput. Phys. 86, 187 (1990).

22. R. Schreiber ANd H. B. Keller, J. Comput. Phys. 49, 165 (1983).

23. W. J. Beyn AND E. J. Doedel, SIAM J. Sci. Statist. Comput. 2, 107 (1981).

24. R. B. KellogG, G. R. Shubin, and A. B. Stephens, SIAM J. Numer. Anal. 17, 733 (1980).

25. A. B. Stephens and G. R. Shubin, SIAM J. Sci. Statist Comput. 2, 404 (1981).

26. G. R. Shubin, A. B. Stephens, and H. M. Glaz, J. Comput. Phys. 39, 364 (1981).

27. A. ISERLES, International Conference on Numerical Mathematics, Singapore, edited by R. P. Agarwal (Birkhauser, Basel, 1989). 
28. A. Iserles and J. M. Sanz-Serna, Numerical Analysis Reports, DAMTP 1989/NA4, May 1989, University of Cambridge, England (unpublished).

29. A. Iserles, A. T. Peplow, ANd A. M. Stuart, DAMTP 1990/NA4, Numerical Analysis Reports, University of Cambridge, March 1990 (unpublished).

30. A. Iserles AND A. M. Stuart, DAMTP 1990/NA6, Numerical Analysis Reports, University of Cambridge, May, 1990 (unpublished).

31. H. C. Yee, P. K. Sweby, ANd D. F. Griffiths, NASA TM-102820, April 1990 (unpublished).

32. R. M. MAY, J. Theoret. Biol. 51, 511 (1975).

33. H. C. YeE, Ph.D. dissertation, University of California Berkeley, CA, 1975.

34. C. S. Hsu, Adv. Appl. Mech. 17, 245 (1977).

35. P. K. SweBY, in Proceedings, Nonlinear Hyperbolic Equations, edited by. J. Ballmann and R. Jeltsch, Notes on Numerical Fluid Mechanics, Vol. 24, 599 (1989).

36. P. K. SweBY, Numerical Analysis Report 6/89, Department of Mathematics, University of Reading, England (unpublished)

37. B. Engquist and B. SJogreen, CAM Report 89-07, Department of Mathematics, UCLA, March 1989 (unmublished).

38. K. W. Wallace, M.S. thesis, Faculty of Science, University of Dundee, Scotland, Sept. 1989 (unpublished).

39. A. M. Panov, Uch. Zap. Ural. Gos. Univ. Vyp. 19, 89 (1956).

40. O. Perron, J. Reine Angew. Math. 161, 41 (1929).

41. C. S. Hsu, H. C. Yee, AND W. H. Cineng, J. Appl. Mech. 44, 147 (1977).

42. C. S. Hsu, H. C. Yee, and W. H. Cheng, J. Sound Vib. 50, 95 (1977).

43. R. M. MAY, Nature 261, 459 (1976).

44. R. M. MAY, Science 186, 645 (1974).

45. T. Y. Li and J. A. Yorke, Am. Math. Monthly 82, 985 (1975).

46. E. N. LORENZ, Tellus 16, 1 (1964).

47. M. J. Feigendaum, J. Statist. Phys. 19, 25 (1978).

48. C. S. Hsu and H. C. Yee, J. Appl. Mech. 44, 870 (1975).

49. S. UshiKi, Physica D 4, 407 (1982).

50. F. BRezzi, S. Ushiki, AND H. FUJI, Numerical Methods for Bifurcation Problems, edited by T. Kupper, H. D. Mittleman, and H. Weber (Birkhauser-Verlag, Boston, 1984).

51. P. G. Reinhall, T. K. Caughey, and D. W. Storti, Trans. ASME, J. Appl. Mech 89-APM-6 (1989).

52. E. N. LoRenz, Physica D 35, 299 (1989).

53. A. J. Lichtenberg and M. A. Lieberman, Regular and Stochastic Motion, Appl. Math. Sci., Bd. 38 (Springer-Verlag, New York, 1938).

54. R. H. Miller, “A Horror Story about Integration Methods," J. Comput. Phys. 93, 469 (1991).

55. W. A. Mulder AND B. van LeER, AIAA-83-1930, July 1983 (unpublished).

56. M. Pruffer, SIAM J. Appl. Math. 45, 32 (1985).

57. W.-J. BEYN, SIAM J. Numer. Anal. 24, 1095 (1987).

58. A. R. Mitchell and D. F. GRIFFiths, Report NA/88 July 1985, Department of Mathematical Sciences, University of Dundee, Scotland, UK (unpublished).

59. A. R. Mitchell and J. C. Bruch, JR., Numer. Methods PDEs 1, 13 (1985).

60. A. R. Mitchell, P. John-Charles, and B. D. Sleeman, Numerical Analysis Report 93, May 1986, Department of Mathematical Sciences, University of Dundee, Scotland (unpublished).

61. V. S. Manoranjan, A. R. Mitchell, and B. D. Sleeman, J. Comput. Appl. Math. 11, 27 (1984).

62. B. D. Sleeman, D. F. Griffiths, A. R. Mitchell, and P. D. Smith, SiaM J. Sci. Statist. Comput. 9, 543 (1988).

63. D. F. Griffiths AND A. R. Mrtchell, Report NA/113, Jan. 1988, Dept. Math. and Comput. Science, University of Dundee, Scotland (unpublished).

64. A. R. Mitchell, G. Stein, and M. Maritz, Commun. Appl. Numer. Methods 4, 263 (1988).

65. D. F. Griffiths AND A. R. Mitchell, Inst. Math. Appl., J. Numer. Anal. 8, 435 (1988). 
66. A. R. Mitchell and S. W. Schoombie, J. Comput. Appl. Math. 25, 363 (1989).

67. J. M. Sanz-Serna ANd F. Vadillo, in Proceedings Dundee, edited by G. A. Watson and D. F. Griffiths (Pitman, London, 1985).

68. A. M. StUART, IMA J. Numer. Anal. 9, 465 (1989).

69. A. Stuart, in Proceedings, NATO Conference on Continuation and Bifurcation, 1989.

70. A. Stuart and A. Peplow, SiaM J. Sci. Statist. Comput., to appear.

71. A. Stuart AND M. S. Floater, European J. Appl. Math., submitted.

72. E. Adams, W. F. Ames, W. Kuhn, and W. Rufeger, Chaos: Computational Errors or Reality, in preparation.

73. E. Adams, IMACS Numer. Appl. Math., edited by W. F. Ames (Baltzer, Basel, 1989), p. 3.

74. E. AdAms, Computer Arithmetic and Self-Validating Numerical Methods (Academic Press, New York, 1990).

75. D. R. Moore N. O. Weiss, AND J. M. Wilkins, Nonlinearity 3, 997 (1990).

76. J. P. KeENer, in Proceedings, IMA Conference on Dynamics of Numerics and Numerics of Dynamics, July 3-August 2, 1990, University of Bristol, Bristol, England.

77. J. SteinhoOf AND A. JAMESON, AIAA J. 20, 1521 (1982).

78. C. Grebogi, E. Ott, AND J. Yorke, Science 238, 585 (1987).

79. S. W. McDonald, C. Grebogi, E. Ott, ANd J. Yorke, Physica D 17, 125 (1985).

80. C. Grebogi, E. OtT, AND J. Yorke, Physica D 7, 181 (1983).

81. J. D. LamberT, Computational Methods in Ordinary Differential Equations (Wiley, New York, 1973).

82. C. W. Gear, Numerical Initial Value Problems in Ordinary Differential Equations (Prentice-Hall, Englewood Cliffs, NJ, 1971).

83. J. C. ButCHER, The Numerical Analysis of Ordinary Differential Equations (Wiley, New York, 1987).

84. MAPLE (algebraic manipulation package), University of Waterloo, Canada, 1988.

85. D. WhitLEY, Bull. London Math. Soc. 15, 177 (1983).

86. R. M. BeAm AND R. F. WARming, "Implicit Numerical Methods for the Compressible Navier-Stokes and Euler Equations," Lecture Notes for Computational Fluid Dynamics, von Karman Institute for Fluid Dynamics, March 29-April 2, 1982, Rhode-Saint-Genese, Belgium (unpublished).

87. G. I. Barenblatt, G. Iooss, AND D. D. JosePH (Eds.), Nonlinear Dynamics and Turbulence, Pitman Advance Publishing Program, Boston, 1983.

88. P. H. Coullet AND E. A. SPiegel, SiaM J. Appl. Math. 43, 776 (1983).

89. T. COAKLEY, AIAA-83-1693, July 1983 (unpublished).

90. J. M. SmITh, Mathematical Ideas in Biology, Cambridge Univ. Press, Cambridge, UK, 1968.

91. G. Strang, SIAM J. Numer. Anal. 5, 506 (1968).

92. R. D. RichtMYer AND K. W. MORTON, Difference Methods for Initial-Value Problems, Interscience-Wiley, New York, 1967.

93. H. IkedA, M. Mimura, AND Y. Nishiura, Nonlinear Anal. Theory, Methods Appl. 13, 507 (1989).

94. T. Hagstrom and H. B. Keller, SIAM J. Sci. Statist. Comput. 7 (1986).

95. J. P. Keener, Pitman Research Notes 157, Ordinary and Partial Differential Equations, edited by B. D. Sleeman and R. J. Jarvis (Longman Sci. Tech., Harlow, 1987), p. 95.

96. P. C. Fife AND J. B. McLeod, Arch. Rat. Mech. Anal. 65, 333 (1977).

97. M. Pandolfi, M. Germano, AND N. Botta, AIAA-88-0514, Jan. 1988 (unpublished).

98. P. Colella, A. Majda, AND V. Roytburd, SIAM J. Sci. Statist. Comput. 7, 1059 (1986).

99. T. R. Young ANd J. P. Boris, J. Phys. Chem. 81, 2424 (1977).

100. H. C. YeE, NASA TM-81265, 1981 (unpublished).

101. H. C. Yee, R. M. Beam, AND R. F. Warming, AIAA J. 20, 1203 (1982).

102. E. Sutanto, "An investigation of the Dynamical Behavior of a Nonlinear Reaction-Advection Equation under Discretization," M.Sc. thesis, University of Reading, 1990 (unpublished).

103. P. D. Lax AND B. Wendroff, Commun. Pure Appl. Math. 13, 217 (1960). 
104. R. J. LeVeque, "Hyperbolic Conservation Laws and Numerical Methods," Lecture Series on Computational Fluid Dynamics, von Karman Institute for Fluid Dynamics, Rhode-Saint-Genese, Belgium, March 5-9, 1990 (unpublished).

105. A. Harten and S. Osher, SIAM J. Numer. Anal. 24, 279 (1987).

106. A. Harten, SIAM J. Numer. Anal. 21, 1 (1984).

107. M. Henon, Commun. Math. Phys. 50, 69 (1976).

108. E. DoEDEL, "AUTO: Software for Continuation and Bifurcation Problems in Ordinary Differential Equations," Cal. Tech. Report, Pasadena, Calif., May, 1986 (unpublished).

109. A. Jameson, W. SChmidt, ANd E. TURKel, AIAA-81-1259, 1981 (unpublished).

110. J. D. ANDERSON, JR., "Introduction to Computational Fluid Dynamics," von Karman Institute for Fluid Dynamics, 1985 Lecture Series, Rhode-Saint-Genese, Belgium (unpublished).

111. G. A. SoD, Numerical Methods in Fluid Dynamics, Cambridge Univ. Press, Cambridge, UK, 1985.

112. J. H. FERzIGER, "Estimation and Reduction of Numerical Error," Forum on Methods of Estimating Uncertainty Limits in Fluid Flow Computations, ASME Winter Annual Meeting, San Francisco, Dec. 1989 (unpublished).

113. P. D. LaX ANd B. Wendroff, Commun. Pure Appl. Math. 17, 381 (1964).

114. R. W. MACCoRmaCK, AIAA-69-354, Cincinnati, Ohio, 1969 (unpublished).

115. N. H. Packard, J. P. Crutchfield, J. D. Farmer, and R. S. Shaw, Phys. Rev. Lett. 45, $712(1980)$.

116. J. P. Eckmann and D. Ruelle, Rev. Mod. Phys. 57, Part I, 617 (1985).

117. H. Froehling, J. P. Crutchfietd, D. Farmer, N. H. Packard, and R: Shaw, Physica D 3, 605 (1981).

118. E. J. Kostelich AND J. A. YorKe, "The Analysis of Experimental Data Using Time-Delay Embedding Methods," Institute for Physical Science and Technology Technical Report, University of Maryland, College Park, MD (unpublished).

119. T. H. Pulliam, AIAA-89-1023, Jan. 9-12, 1989 (unpublished).

120. T. H. Pulliam, "Numerical Simulation of Chaotic Flows: Measures of Chaos," in Proceedings, Forum on Chaotic Dynamics in Fluid Dynamics, ASME Fluids Engineering Spring Conference, La Jolla, July 1989, pp. 145-151 (unpublished).

121. A. Fortin, M. Fortin, and J. J. Gervais, J. Comput. Phys. 70, 295 (1987). 